\title{
The Influence of Shaft's Bending on the Coupling Vibration of a Flexible Blade-Rotor System
}

\author{
Chao-feng Li, Hou-xin She, Wen Liu, and Bang-chun Wen \\ School of Mechanical Engineering and Automation, Northeastern University, Shenyang, Liaoning 110819, China \\ Correspondence should be addressed to Chao-feng Li; chaof.li@gmail.com
}

Received 28 May 2016; Revised 14 December 2016; Accepted 16 February 2017; Published 19 March 2017

Academic Editor: Dane Quinn

Copyright (C) 2017 Chao-feng Li et al. This is an open access article distributed under the Creative Commons Attribution License, which permits unrestricted use, distribution, and reproduction in any medium, provided the original work is properly cited.

\begin{abstract}
The influence of shaft bending on the coupling vibration of rotor-blades system is nonignorable. Therefore, this paper analyzed the influence of shaft bending on the coupling vibration of rotor-blades system. The vibration mode function of shaft under elastic supporting condition was also derived to ensure accuracy of the model as well. The influence of the number of blades, the position of disk, and the support stiffness of shaft on critical speed of system was analyzed. The numerical results show that there were two categories of coupling mode shapes which belong to a set where the blade's first two modes predominate in the system: shaft-blade (SB) mode and interblade (BB) mode due to the coupling between blade and shaft. The BB mode was of repeated frequencies of $\left(N_{b}-2\right)$ multiplicity for number blades, and the SB mode was of repeated frequencies of (2) multiplicity for number blades. What is more, with the increase of the number of blades, natural frequency of rotor was decreasing linearly, that of BB mode was constant, and that of SB mode was increasing linearly. Natural frequency of BB mode was not affected while that of rotor and SB mode was affected (changed symmetrically with the center of shaft) by the position of disk. In the end, vibration characteristics of coupling mode shapes were analyzed.
\end{abstract}

\section{Introduction}

In the compressor/turbine engineering equipment, the blade, the disk, and the shaft are assembled together by a certain connecting structure and forming a blade-disk-shaft coupling system which has some coupling characteristics. With the development of scientific research, many scholars in this field have carried out a lot of research on the vibration of blades rotor coupling system. However, the influence of shaft bending on the coupling vibration of rotor-blades system is nonignorable. And there are potential couplings between shaft bending and blade bending under the right condition in fact. Therefore, this present research focuses on the influence of shaft bending on the coupling vibration of rotor-blades system.

The flexible disk model is the most popular analytical model for such systems at first. Parker and Mote [1] predicted natural frequencies of stationary annular or circular plates with a perturbation solution, and they discussed the mode split of the repeated natural frequencies. Khorasany and
Hutton [2] studied linear vibration behavior of annular spinning disk whose inner boundary is free to move in the axial direction while the body of the disk is constrained by a space fixed linear spring. Kim et al. [3] reported that significant changes could occur to its natural frequencies and modes when a structure deviates from axisymmetry because of circumferentially varying model features. Dopkin and Shoup [4] studied the influence of disk's flexibility on natural frequencies by a transfer matrix method.

As the rotating systems become lighter and more flexible with higher operating speed for higher productivity and economical design, the rigid shaft-disk model cannot accurately predict the vibration characteristics of the system. And the shaft-blades model is considered, considering the coupling vibration among shaft bending and blade bending. Anegawa et al. [5] successfully analyzed the coupling of the shaft with in-plane vibration of the blades with $k=1$ modes in a 1-DOF mass-spring system. What is more, the existence of an unstable region due to coupling and the characteristics of coupled resonance were indicated which 
was confirmed by experimental data. Turhan and Bulut [6] studied coupling effects between shaft-torsional and bladebending (in-plane) vibrations in turbomachinery and other rotating bladed structures and considered an idealized model where the blades are represented by uniform Euler-Bernoulli beams. Besides, they derived a linearized mathematical model for the system by using synthetically, multiframe, and mixed (consisting in a coalescence of finite element and Galerkin methods) approach. Chiu and Chen [7] investigated the influence on coupling vibrations among shaft-torsion and blade-bending coupling vibrations of a multidisk rotor system analytically. They solved the natural frequencies and the mode shapes of the system for one- to three-disk cases as examples and drew the diagrams of the coupling mode shapes. From the results, it was found that the interblade (BB) modes were of repeated frequencies of $\left(N_{b}-1\right)$ multiplicity for number of blades. What is more, the numerical calculations also revealed that the natural frequencies were affected by blade numbers and disk distance. Lesaffre et al. [8] presented a model of fully flexible bladed rotor developed in the rotating frame and took the gyroscopic effects as well as the spin softening effects and the centrifugal stiffening effects into consideration. Huang and Ho [9] introduced a new approach to analyzing the dynamic coupling between shaft torsion and blade bending of a rotating shaft-disk-blade unit. Numerical examples showed not only coupling between the shaft, disk, and blades, but also coupling between individual blades and the blade coupling modes occurred only in repeated frequencies. What is more, the effect of shaft speed on the modal frequencies was investigated. Plots illustrating the occurrence of critical speeds and flutter instabilities are presented. Chiu and Yang [10] investigated the influence on coupling vibrations among shaft-torsion, blade-bending, and lacing wire coupling vibrations of a multidisk rotor system with grouped blades analytically. Moreover, they also found that not only the natural frequencies in a mistuned blade but also the types of coupling modes were found changed.

Then the disk-blade model is used to predict the influence of stagger and twist on natural frequencies for stationary as well as rotating bladed disk systems [11]. And the flexible disk-blades model attracts a lot of research since the early researches [12-14]. To get a better understanding and an accurate prediction of the dynamic characteristics of shaftdisk-blade units, Chun and Lee [15] developed an analysis substructure synthesis and assumed modes method to investigate the effect of the flexibility of a bladed disk assembly on the vibrational modes of a flexible rotor system. Yang and Huang [16] studied the longitudinal motion effects on the coupled vibration in a shaft-disk-blades system. Yang and Huang [17-19] investigated the coupling vibrations and applications among support-longitudinal, shaft-torsion, disktransverse, and blade-bending vibrations in a shaft-diskblades unit. Chiu and Huang [20] investigated the influence on coupling vibrations among shaft torsion, disk-transverse, and blade bending of a rotor system with a mistuned blade length analytically and found that the shaft-disk-blades system appeared to have four types of coupling vibrations: shaft-blade (SB), shaft-disk-blade (SDB), disk-blade (DB), and blade-blade (BB) mode.
From the preceding research it could be seen that there were two kinds of analysis method for the typical structure. One was regarding the support of blade as clamped or elastic support and analyzing the vibration of the blade separately and the other was regarding blade-disk system as a rigid body component and analyzing the dynamic characteristics of the rotor system separately. However, the research on blade-disk-shaft coupling system had gradually become the focus of research. Although some scholars had researched the coupling vibration of shaft torsion and blade bending, the influence of shaft's bending is usually ignored. But we found that there is a new mode which is SB due to the coupling vibration of shaft's bending and blade's bending. And the present paper aim is to analyze the influence of shaft bending on the coupling vibration of shaft-disk-blades system. What is more, influences of the blades number, disk position, and support stiffness on the characteristic value of system, the Campbell diagram, and the mode shape of vibration were studied by numerical simulation.

\section{The Establishment of Dynamic Model}

A dynamic model of rotor-blade coupling system with elastic restraints is shown in Figure 1. The rotor system is composed of continuous flexible shaft and rigid disk. The Timoshenko beam model is used to derive the energy equation of shaft and the bending and torsional vibration are also taken into consideration. The disk is regarded as a mass point. Considering the radial and transverse vibration of blade, the energy equation of blade is derived by using the EulerBernoulli beam model. $O-X Y Z$ is the global coordinate system. $o_{r}-x_{r} y_{r} z_{r}$ is the local coordinate system of disk where the geometric center of disk is the origin. $o_{b}-x_{b} y_{b} z_{b}$ is the local coordinate system of blade where the geometric center of blade root is the origin, and $o$ point is an arbitrary point on the blade. As shown in the figure, both ends of the rotor are elastic supported, where the elastic support stiffness and damping at both ends are assumed to be equal.

\subsection{The Establishment of Flexible Rotor Model}

2.1.1. The Establishment of the Energy Equation of Flexible Rotor Model. According to the relevant knowledge of elastic mechanics, the kinetic energy of the shaft can be expressed as

$$
\begin{aligned}
T_{s} & =\frac{1}{2} \int_{0}^{L_{s}}\left(\rho A\left(\dot{x}^{2}+\dot{y}^{2}\right)+I_{s}\left(\frac{\partial \theta_{T}}{\partial t}+\Omega\right)^{2}+J_{p} \dot{\phi}^{2}\right. \\
& \left.+J_{p} \dot{\phi}\left(\dot{\theta}_{x} \theta_{y}-\dot{\theta}_{y} \theta_{x}\right)+J_{d}\left(\dot{\theta}_{x}^{2}+\dot{\theta}_{y}{ }^{2}\right)\right) \mathrm{d} z,
\end{aligned}
$$

where $J_{d}$ and $J_{p}$ are cross-sectional moment of inertia (transverse moment of inertia) and polar moment of inertia, respectively.

Regarding the disk as a mass point and ignoring the influence of the disk on the vibration mode of beam, the 


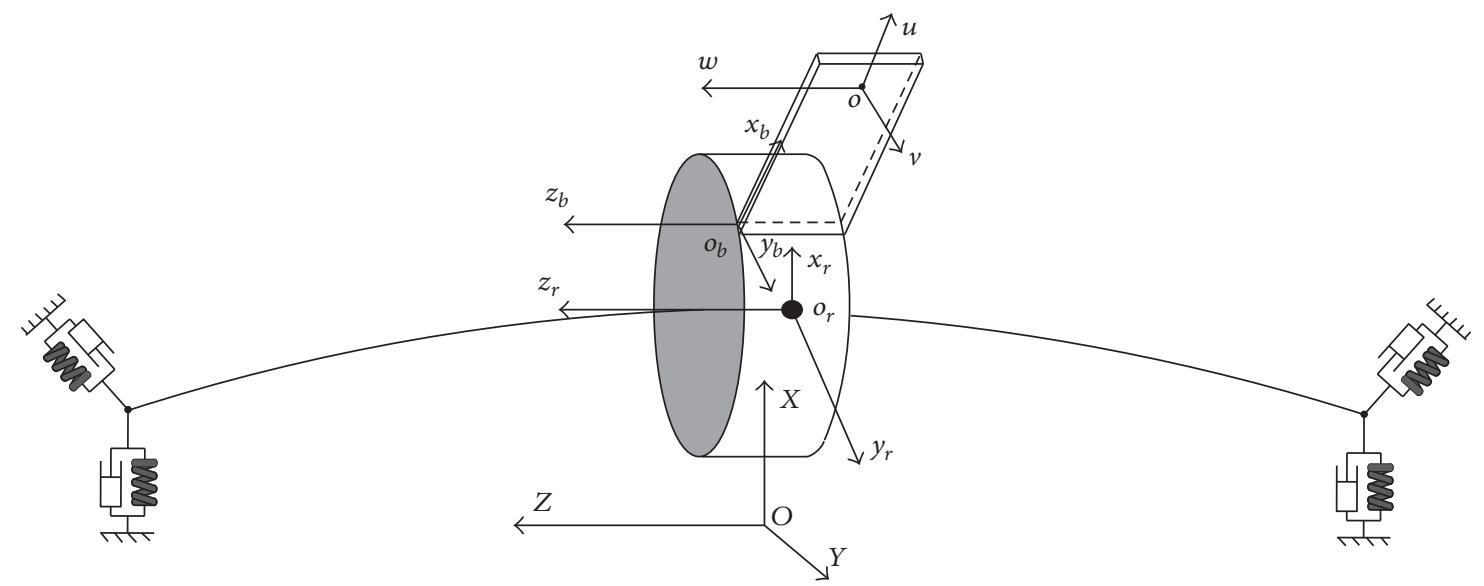

FIGURE 1: Schematic diagram of the model of rotor-blade coupling system.

kinetic energy of disk can be represented in the following form:

$$
\begin{aligned}
T_{d}= & \frac{1}{2} m_{d} \dot{x}_{d}^{2}+\frac{1}{2} m_{d} \dot{y}_{d}^{2}+\frac{1}{2} J_{d z} \dot{\theta}_{x}^{2}+\frac{1}{2} J_{d z} \dot{\theta}_{y}^{2} \\
& +\frac{1}{2} J_{z} \dot{\phi}\left(\dot{\theta}_{x} \theta_{y}-\dot{\theta}_{y} \theta_{x}\right)+\frac{1}{2} J_{z} w^{2},
\end{aligned}
$$

where $m_{d}$ is the mass of disk and $z_{d}$ is the distance between the position of disk and left end point of shaft.

The total kinetic energy of rotor system can be obtained as follows:

$$
T_{r}=T_{d}+T_{s}
$$

Analyzing the model of shaft and considering the torsion, bending, and shearing, the total potential energy of shaft can be given as follows:

$$
\begin{aligned}
U_{s} & =\frac{1}{2} \int_{0}^{L_{s}} G_{s} J_{s}\left(\frac{\partial \theta_{T}}{\partial t}\right)^{2} \\
& +\left\{E_{s} I_{s}\left[\left(\frac{\partial^{2} x}{\partial z^{2}}\right)^{2}+\left(\frac{\partial^{2} y}{\partial z^{2}}\right)^{2}\right]\right. \\
& \left.+\kappa_{s} G_{s} A_{s}\left[\left(\frac{\partial x}{\partial z}-\theta_{x}\right)^{2}+\left(\frac{\partial y}{\partial z}-\theta_{y}\right)^{2}\right]\right\} \mathrm{d} z,
\end{aligned}
$$

where $G_{s}, E_{s}, I_{s}, \kappa_{s}$, and $A_{s}$ denote the shaft's shear elastic modulus, elastic modulus, polar rotary inertia, torsional rigidity, and cross-sectional area, respectively.

\subsubsection{The Vibration Mode Function of Shaft with Elastic} Support. In order to simulate the boundary condition of shaft more accurately, the shaft is simplified as an elastic support beam with springs at both ends and simplified model is shown in Figure 2, the span and mass of uniform vertical elastic support beam $A B$ are $l$ and $m$, respectively, and the stiffness of spring at $A$ and $B$ ends is $k_{1}$ and $k_{2}$, respectively.

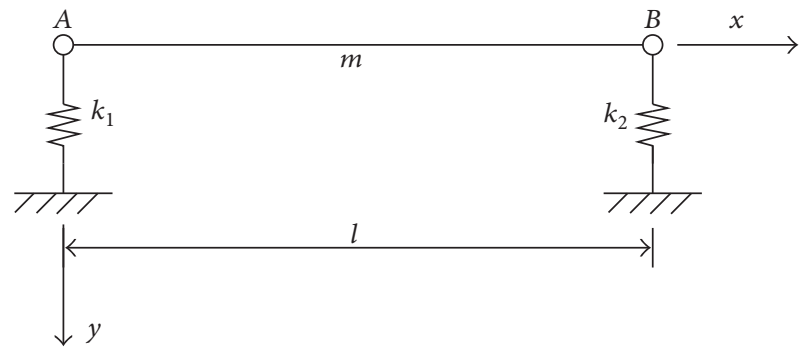

FIgURE 2: Schematic diagram of the simplified elastic support beam model.

Assuming the central axis of each section of beam has bending vibration at the same plane, applicating plane assumption in the process of vibration, excluding the effects of rotational inertia and shear deformation, and ignoring the rotation around the center axis of the cross-section and damping, the free vibration differential equation of beam has the following expression:

$$
m \frac{\partial^{2} y(x, t)}{\partial t^{2}}+\frac{\partial^{2}}{\partial x^{2}}\left(E I \frac{\partial^{2} y(x, t)}{\partial x^{2}}\right)=0,
$$

where $y(x, t)$ donate the transverse displacement of the beam, and it is the function of two variables including the position of the section $x$ and the time $t$.

The solution of (5) is separated from space and time. Assumed modes method is used to discretize shaft deformation, so the beam transverse displacement $y(x, t)$ can be written as follows:

$$
y(x, t)=Y(x) F(t),
$$

where $Y(x)$ is vibration mode function. Substituting (6) into (5), the differential equation of beam can be gotten:

$$
\frac{\mathrm{d}^{4} Y(x)}{\mathrm{d} x^{4}}-\beta^{4} Y(x)=0,
$$


where

$$
\beta^{4}=\frac{\omega^{2} m}{E I} .
$$

Equation (7) is a four-order linear ordinary differential equation with constant coefficients; it can be solved by using Euler transformation

$$
Y(x)=C_{1} \sin \beta x+C_{2} \cos \beta x+C_{3} \operatorname{sh} \beta x+C_{4} \operatorname{ch} \beta x,
$$

where $\omega$ is the natural frequency of beam and $C_{1}, C_{2}, C_{3}$, and $\mathrm{C}_{4}$ are integral constants.

As the elastic support beam is adopted, boundary conditions can be expressed as

$$
\begin{array}{ll}
A \text { end: } & Y^{\prime \prime}(0)=0 \\
& E I Y^{\prime \prime \prime}(0)=-k_{1} Y(0)
\end{array}
$$

$B$ end: $\quad Y^{\prime \prime}(l)=0$

$$
E I Y^{\prime \prime \prime}(l)=-k_{2} Y(l) .
$$

Assuming $\lambda=\beta l$ and substituting (10) into (9), we can get the equation about $C_{2}$ and $C_{4}$ :

$$
C_{2}=C_{4}
$$

Substituting (11) and (12) into (9), then

$$
\left[\begin{array}{ccc}
\frac{E I \lambda^{3}}{2 k_{1} l^{3}} & -1 & -\frac{E I \lambda^{3}}{2 k_{1} l^{3}} \\
-\sin \lambda & -\cos \lambda+\operatorname{ch} \lambda & \operatorname{sh} \lambda \\
\sin \lambda+\frac{E I \lambda^{3}}{2 k_{2} l^{3}} \cos \lambda & \cos \lambda+\operatorname{ch} \lambda-\frac{E I \lambda^{3}}{2 k_{2} l^{3}}(\sin \lambda+\operatorname{sh} \lambda) & \operatorname{sh} \lambda-\frac{E I \lambda^{3}}{2 k_{2} l^{3}} \operatorname{ch} \lambda
\end{array}\right]\left[\begin{array}{l}
C_{1} \\
C_{2} \\
C_{3}
\end{array}\right]=0
$$

If there are nonzero solutions for $C_{1}, C_{2}$, and $C_{3}$, the determinant of the coefficients must be zero in (13). The root is obtained by the method of numerical solution, and then substituting the root and (12) into (9), the vibration mode function of elastic support beam can be written as

$$
\begin{aligned}
& Y(x)=\cosh \frac{\lambda_{i}}{l} x+\cos \frac{\lambda_{i}}{l} x+2 \frac{k_{1} l^{3}}{E I \lambda_{i}^{3}} \cdot \sin \frac{\lambda_{i}}{l} x \\
& +\frac{2 k_{1} l^{3} / E I \lambda_{i}^{3}+\cos \lambda_{i}+\cosh \lambda_{i}}{\sinh \lambda_{i}-\sin \lambda_{i}}\left(\sinh \frac{\lambda_{i}}{l} x\right. \\
& \left.+\sin \frac{\lambda_{i}}{l} x\right),
\end{aligned}
$$

where $E$ and $I$ are Young modulus and moment of inertia of shaft, assuming $k_{1}=k_{2}$.

2.1.3. Discretization of the Energy Equation of the Elastic Rotor Model. Vibration model functions of the direction of $x, y$, and torsion are $X_{i}(z), Y_{i}(z)$, and $\Phi_{i}$, respectively. Introducing regular coordinates $\eta_{i}(t), \xi_{i}(t)$, and $q_{\theta}$. The assumed mode method is adopted to discretize the continuous system: that is,

$$
\theta_{T}(z, t)=\sum_{i=1}^{\infty} \Phi_{i}(z) q_{\theta i}(t)=\Phi(z) \mathbf{q}_{\theta}(t)
$$

Assuming that the shear force $Q$ is constant, the bending angle obtained as follows:

$$
\begin{aligned}
\theta_{x}(z, t) & =x^{\prime}+\frac{E I}{\kappa A G} x^{\prime \prime \prime} \\
& =\mathbf{X}^{\prime}(z) \boldsymbol{\eta}(t)+\frac{E I}{\kappa A G} \mathbf{X}^{\prime \prime \prime}(z) \boldsymbol{\eta}(t) \\
\theta_{y}(z, t) & =y^{\prime}+\frac{E I}{\kappa A G} y^{\prime \prime \prime} \\
& =\mathbf{Y}^{\prime}(z) \xi(t)+\frac{E I}{\kappa A G} \mathbf{Y}^{\prime \prime \prime}(z) \xi(t) .
\end{aligned}
$$

Substituting (15) (17) into (3), the discrete kinetic energy of shaft is shown in Appendix A.

Ignoring the member does not appear in the equation; it is written as follows:

$$
\begin{aligned}
T_{s}= & \frac{1}{2}\left[\begin{array}{lll}
\dot{\boldsymbol{\eta}}(t) & \dot{\boldsymbol{\xi}}(t) & \dot{\mathbf{q}}_{\theta}
\end{array}\right]\left[\begin{array}{ccc}
\mathbf{M}_{s 1} & \mathbf{0} & \mathbf{0} \\
\mathbf{0} & \mathbf{M}_{s 2} & \mathbf{0} \\
\mathbf{0} & \mathbf{0} & \mathbf{M}_{\boldsymbol{\theta}}
\end{array}\right]\left[\begin{array}{c}
\dot{\boldsymbol{\eta}}(t) \\
\dot{\boldsymbol{\xi}}(t) \\
\dot{\mathbf{q}}_{\boldsymbol{\theta}}
\end{array}\right] \\
& +\frac{1}{2}\left[\begin{array}{llll}
\dot{\boldsymbol{\eta}}(t) & \dot{\boldsymbol{\xi}}(t) & \dot{\mathbf{q}}_{\boldsymbol{\theta}}
\end{array}\right] \dot{\phi}\left[\begin{array}{ccc}
\mathbf{0} & \mathbf{G}_{s 1} & \mathbf{0} \\
\mathbf{G}_{s 2} & \mathbf{0} & \mathbf{0} \\
\mathbf{0} & \mathbf{0} & \mathbf{0}
\end{array}\right]\left[\begin{array}{c}
\boldsymbol{\eta}(t) \\
\boldsymbol{\xi}(t) \\
\mathbf{q}_{\boldsymbol{\theta}}
\end{array}\right] .
\end{aligned}
$$

$$
\begin{aligned}
& x(z, t)=\sum_{i=1}^{\infty} X_{i}(z) \eta_{i}(t)=\mathbf{X}(z) \boldsymbol{\eta}(t) \\
& y(z, t)=\sum_{i=1}^{\infty} Y_{i}(z) \xi_{i}(t)=\mathbf{Y}(z) \boldsymbol{\xi}(t)
\end{aligned}
$$


Discretizing the kinetic energy of disk, the discrete kinetic energy of disk is shown in Appendix A.

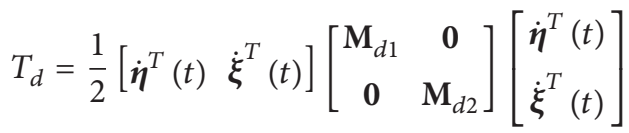

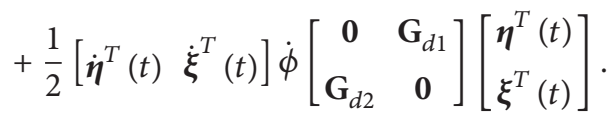

The total kinetic energy of the rotor system is

$$
\begin{aligned}
T_{r} & =T_{s}+T_{d}=\frac{1}{2} \\
& \cdot\left[\begin{array}{lll}
\dot{\boldsymbol{\eta}}(t) & \dot{\boldsymbol{\xi}}(t) & \dot{\mathbf{q}}_{\boldsymbol{\theta}}
\end{array}\right]\left[\begin{array}{ccc}
\mathbf{M}_{s 1}+\mathbf{M}_{d 1} & \mathbf{0} & \mathbf{0} \\
\mathbf{0} & \mathbf{M}_{s 2}+\mathbf{M}_{d 2} & \mathbf{0} \\
\mathbf{0} & \mathbf{0} & \mathbf{M}_{\boldsymbol{\theta}}
\end{array}\right]\left[\begin{array}{c}
\dot{\boldsymbol{\eta}}(t) \\
\dot{\boldsymbol{\xi}}(t) \\
\dot{\mathbf{q}}_{\boldsymbol{\theta}}
\end{array}\right] \\
& +\frac{1}{2} \\
& \cdot\left[\begin{array}{lll}
\dot{\boldsymbol{\eta}}(t) & \dot{\boldsymbol{\xi}}(t) & \dot{\mathbf{q}}_{\boldsymbol{\theta}}
\end{array}\right] \dot{\phi}\left[\begin{array}{ccc}
\mathbf{0} & \mathbf{G}_{s 1}+\mathbf{G}_{d 1} & \mathbf{0} \\
\mathbf{G}_{s 2}+\mathbf{G}_{d 2} & \mathbf{0} & \mathbf{0} \\
\mathbf{0} & \mathbf{0} & \mathbf{0}
\end{array}\right]\left[\begin{array}{c}
\boldsymbol{\eta}(t) \\
\boldsymbol{\xi}(t) \\
\mathbf{q}_{\boldsymbol{\theta}}
\end{array}\right] .
\end{aligned}
$$

In the same way, discretizing the potential energy of shaft, the discrete potential energy of shaft is shown in Appendix A.

In order to facilitate the representation and computation, it is written as

$$
U_{s}=\frac{1}{2}\left[\begin{array}{lll}
\boldsymbol{\eta}(t) & \boldsymbol{\xi}(t) & \mathbf{q}_{\boldsymbol{\theta}}
\end{array}\right]\left[\begin{array}{ccc}
\mathbf{K}_{s 1} & \mathbf{0} & \mathbf{0} \\
\mathbf{0} & \mathbf{K}_{s 2} & \mathbf{0} \\
\mathbf{0} & \mathbf{0} & \mathbf{K}_{\boldsymbol{\theta}}
\end{array}\right]\left[\begin{array}{c}
\boldsymbol{\eta}(t) \\
\boldsymbol{\xi}(t) \\
\mathbf{q}_{\boldsymbol{\theta}}
\end{array}\right] .
$$

Substituting the kinetic energy equation (20) and potential energy equation (21) into the Lagrange equations yields the discretized equations of motion in matrix notation as

$$
\mathbf{M}_{r} \ddot{\mathbf{q}}_{r}+\left(\mathbf{C}_{r}+\dot{\phi} \mathbf{G}_{r}\right) \dot{\mathbf{q}}_{r}+\mathbf{K}_{r} \mathbf{q}_{r}=0,
$$

where matrices $\mathbf{M}_{r}, \mathbf{C}_{r}, \mathbf{G}_{r}$, and $\mathbf{K}_{r}$ are mass, damping, gyroscopic, and stiffness matrices of the rotor system,

$$
\begin{aligned}
& \mathbf{M}_{r}=\left[\begin{array}{ccr}
\mathbf{M}_{s 1}+\mathbf{M}_{d 1} & \mathbf{0} & \mathbf{0} \\
\mathbf{0} & \mathbf{M}_{s 2}+\mathbf{M}_{d 2} & \mathbf{0} \\
\mathbf{0} & \mathbf{0} & \mathbf{M}_{\boldsymbol{\theta}}
\end{array}\right], \\
& \mathbf{C}_{r}=\left[\begin{array}{ccc}
\mathbf{C}_{s 1}+\mathbf{C}_{d 1} & 0 & 0 \\
0 & \mathbf{C}_{s 2}+\mathbf{C}_{d 2} & 0 \\
0 & 0 & \mathbf{C}_{\boldsymbol{\theta}}
\end{array}\right], \\
& \mathbf{G}_{r}=\left[\begin{array}{ccc}
0 & \mathbf{G}_{s 1}+\mathbf{G}_{d 1} & 0 \\
\mathbf{G}_{s 2}+\mathbf{G}_{d 2} & 0 & 0 \\
0 & 0 & 0
\end{array}\right], \\
& \mathbf{K}_{r}=\left[\begin{array}{ccc}
\mathbf{K}_{s 1} & 0 & 0 \\
0 & \mathbf{K}_{s 2} & 0 \\
0 & 0 & \mathbf{K}_{\boldsymbol{\theta}}
\end{array}\right],
\end{aligned}
$$

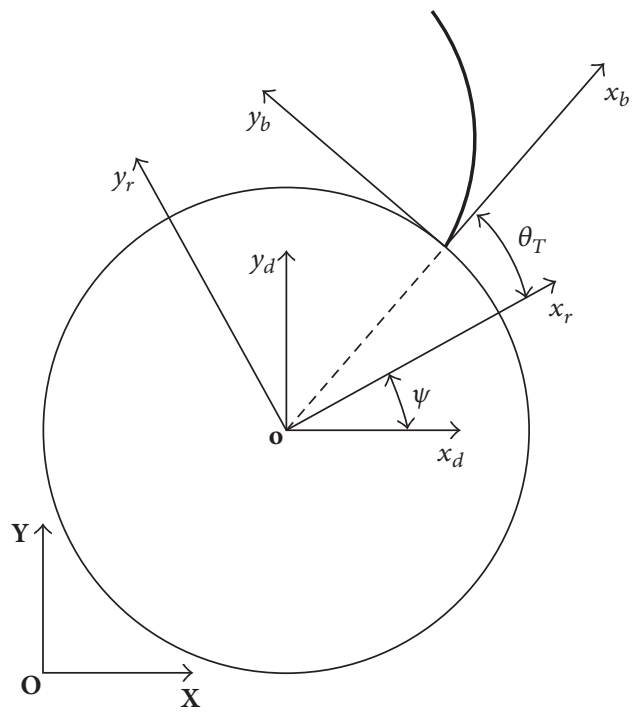

FIGURE 3: Schematic diagram of disk-blade system.

and the meaning of specific parameters is shown in Appendix A, where matrices $\mathbf{M}_{r}, \mathbf{C}_{r}, \mathbf{G}_{r}$, and $\mathbf{K}_{r}$ are mass, damping, gyroscopic, and stiffness matrices of the rotor system.

2.2. The Establishment of Energy Equation of Rotating Blade. Assuming there are $N_{b}$ blades evenly distributed on the rigid disk, so the disk-blade system is a circular symmetrical structure. When the $i$ th blade is deformed, the schematic diagram of disk-blade system is shown in Figure 3.

The displacement of arbitrary point $Q$ on the blade in the fixed coordinate system can be written as

$$
\begin{aligned}
& \mathbf{r}_{\mathrm{Q}}=\left[\begin{array}{lll}
x_{d} & y_{d} & z_{d}
\end{array}\right]^{T} \\
& +\mathbf{A}_{0} \mathbf{A}_{1} \mathbf{A}_{2} \mathbf{A}_{3}[(R+x+u) \quad v \quad w]^{T},
\end{aligned}
$$

where $x_{d}, y_{d}$, and $z_{d}$ are the displacement of $X, Y$, and $Z$ direction of the disk in the global coordinate system. $\mathbf{A}_{0}$ and $\mathbf{A}_{1}$ are the transformation matrix from local coordinate system to the rotational coordinate system of the blade. $\mathbf{A}_{2}$ is the transformation matrix from rotating coordinate system to the swinging coordinate system. $\mathbf{A}_{3}$ is the transformation matrix of the coordinate system caused by the swing. The specific meaning is given in Appendix B.

When $N_{b}>3$, the circular symmetric structure has good geometrical and mechanical properties. Without taking into account the axial motion of rotor, the kinetic energy of the $i$ th blade is

$$
T_{b}=\frac{1}{2} \rho_{b} A_{b} \int_{0}^{L_{b}} \dot{\mathbf{r}}_{Q}^{2} \mathrm{~d} x .
$$


Specific expression is as follows:

$$
\begin{aligned}
T_{b} & =\frac{1}{2} \rho_{b} A_{b} \int_{0}^{L_{b}}\left(\dot{x}_{d}^{2}+\dot{y}_{d}^{2}+\dot{u}^{2}+\dot{v}^{2}+\dot{\psi}^{2}\left(u^{2}+v^{2}\right)\right. \\
& +(R+x)^{2}\left(\dot{\theta}_{T}^{2}+\dot{\psi}^{2} \theta_{T}^{2}+\dot{\theta}_{x}^{2}\right)-2 \dot{u} v \dot{\psi}+2 u \dot{v} \dot{\psi} \\
& +2 \dot{x}_{d} \dot{u} \cos \psi-2 \dot{x}_{d} \dot{v} \sin \psi+2 \dot{y}_{d} \dot{u} \sin \psi \\
& +2 \dot{y}_{d} \dot{v} \cos \psi-2(R+x) \dot{x}_{d} \dot{\theta}_{T} \sin \psi \\
& +2(R+x) \dot{y}_{d} \dot{\theta}_{T} \cos \psi+2(R+x) \dot{v} \dot{\theta}_{T} \\
& -2 \dot{x}_{d} u \dot{\psi} \sin \psi-2 \dot{x}_{d} v \dot{\psi} \cos \psi+2 \dot{y}_{d} u \dot{\psi} \cos \psi \\
& -2 \dot{y}_{d} v \dot{\psi} \sin \psi-2(R+x) \dot{x}_{d} \theta_{T} \dot{\psi} \cos \psi \\
& \left.-2(R+x) \dot{y}_{d} \theta_{T} \dot{\psi} \sin \psi+4(R+x) u \dot{\theta}_{T} \dot{\psi}\right) \mathrm{d} x,
\end{aligned}
$$

where $\rho_{b}, A_{b}$, and $L_{b}$ are density, cross-sectional area, and length of the blade, respectively; $R$ is radius of disk; $\psi=$ $\Omega t+(i-1) 2 \pi / N_{b} ; \theta_{T}$ is the twist angle at disk hub.

Considering the effects of bending, circumferential compression, and centrifugal and normal-force-generating potential energy of blade, total potential energy of $i$ th blade can be expressed as

$$
\begin{aligned}
U_{b} & =\frac{1}{2} E_{b} A_{b} \int_{0}^{L_{b}}\left(\frac{\partial u}{\partial x}\right)^{2} \mathrm{~d} x+\frac{1}{2} \\
& \cdot E_{b} I_{b} \int_{0}^{L_{b}}\left(\frac{\partial^{2} v}{\partial x^{2}}\right)^{2} \mathrm{~d} x+\frac{1}{4} \\
\cdot & \rho_{b} A_{b} \dot{\psi}^{2} \int_{0}^{L_{b}}\left[L_{b}^{2}-x^{2}+2 R\left(L_{b}-x\right)\right]\left(\frac{\partial v}{\partial x}\right)^{2} \mathrm{~d} x
\end{aligned}
$$

where $E_{b}$ and $I_{b}$ are Young's modulus and cross-section area moment of inertia of blade.

Because the movement of end of the rigid disk and the blade is consistent, the modal function of cantilever beam is chosen for blade:

$$
\begin{aligned}
\phi_{i}(x)= & V_{i}(x) \\
= & \cosh \frac{\lambda_{i}}{l} x-\cos \frac{\lambda_{i}}{l} x \\
& -\frac{\cosh \lambda_{i}+\cos \lambda_{i}}{\sinh \lambda_{i}+\sin \lambda_{i}}\left(\sinh \frac{\lambda_{i}}{l} x-\sin \frac{\lambda_{i}}{l} x\right) .
\end{aligned}
$$

Vibration model functions of the displacement of blades $u$ and $v$ are $\phi_{i}(x)$ and $V_{i}(x)$. The assumed mode method is adopted to discretize the continuous system: that is,

$$
\begin{aligned}
& u=\sum_{i=1}^{n} \phi_{i}(x) q_{u i}=\boldsymbol{\Phi} \mathbf{q}_{\mathbf{u}} \\
& v=\sum_{i=1}^{n} V_{i}(x) q_{v i}=\Psi \mathbf{q}_{\mathbf{v}} .
\end{aligned}
$$

Substituting (29) into (26) and (27), the discrete energy equation of $i$ th blade is given by

$$
\begin{aligned}
& T_{b}=\frac{1}{2} \rho_{b} A_{b} \int_{0}^{L_{b}} \dot{\boldsymbol{\eta}}^{\mathrm{T}} \mathbf{X}^{\mathrm{T}}\left(z_{d}\right) \mathbf{X}\left(z_{d}\right) \dot{\boldsymbol{\eta}}+\dot{\boldsymbol{\xi}}^{\mathrm{T}} \mathbf{Y}^{\mathrm{T}}\left(z_{d}\right) \\
& \cdot \mathbf{Y}^{\mathrm{T}}\left(z_{d}\right) \dot{\boldsymbol{\xi}}+\dot{\mathbf{q}}_{\mathbf{u}}^{\mathrm{T}} \boldsymbol{\Psi}^{\mathrm{T}} \boldsymbol{\Psi} \dot{\mathbf{q}}_{\mathbf{u}}+\dot{\mathbf{q}}_{\mathbf{u}}^{\mathrm{T}} \Psi^{\mathrm{T}} \boldsymbol{\Psi} \dot{\mathbf{q}}_{\mathbf{u}}+\dot{\mathbf{q}}_{\mathbf{v}}^{\mathrm{T}} \mathbf{V}^{\mathrm{T}} \mathbf{V} \dot{\mathbf{q}}_{\mathbf{v}} \\
& +\dot{\mathbf{q}}_{\boldsymbol{\theta}}^{\mathrm{T}}(R+x)^{2} \boldsymbol{\Phi}^{\mathrm{T}} \boldsymbol{\Phi} \dot{\mathbf{q}}_{\boldsymbol{\theta}}+\mathbf{q}_{\mathbf{u}}^{\mathrm{T}} \dot{\psi}^{2} \boldsymbol{\Psi}^{\mathrm{T}} \boldsymbol{\Psi} \mathbf{q}_{\mathbf{u}} \\
& +\mathbf{q}_{\mathbf{v}}^{\mathrm{T}} \dot{\psi}^{2} \mathbf{V}^{\mathrm{T}} \mathbf{V} \mathbf{q}_{\mathbf{v}}+\mathbf{q}_{\boldsymbol{\theta}}^{\mathrm{T}}(R+x)^{2} \dot{\psi}^{2} \boldsymbol{\Phi}^{\mathrm{T}} \boldsymbol{\Phi} \mathbf{q}_{\boldsymbol{\theta}} \\
& -2 \dot{\boldsymbol{\eta}}^{\mathrm{T}} \mathbf{X}^{\mathrm{T}} \mathbf{V} \sin \psi \dot{\mathbf{q}}_{\mathbf{v}}+2 \dot{\mathbf{q}}_{\mathbf{v}}^{\mathrm{T}}(R+x) \mathbf{V}^{\mathrm{T}} \boldsymbol{\Phi} \dot{\mathbf{q}}_{\boldsymbol{\theta}} \\
& +2 \dot{\boldsymbol{\eta}}^{\mathrm{T}} \mathbf{X}^{\mathrm{T}}\left(z_{d}\right) \Psi \cos \psi \dot{\mathbf{q}}_{\mathbf{u}}+2 \dot{\boldsymbol{\xi}}^{\mathrm{T}} \mathbf{Y}^{\mathrm{T}}\left(z_{d}\right) \Psi \sin \psi \dot{\mathbf{q}}_{\mathbf{u}} \\
& -2 \dot{\boldsymbol{\eta}}^{\mathrm{T}}(R+x) \mathbf{X}^{\mathrm{T}}\left(z_{d}\right) \boldsymbol{\Phi} \sin \psi \dot{\mathbf{q}}_{\boldsymbol{\theta}}+2 \dot{\boldsymbol{\xi}}^{\mathrm{T}}(R+x) \\
& \cdot \mathbf{Y}^{\mathrm{T}}\left(z_{d}\right) \boldsymbol{\Phi} \cos \psi \dot{\mathbf{q}}_{\boldsymbol{\theta}}+\dot{\boldsymbol{\eta}}^{\mathrm{T}}(R+x)^{2} \\
& \cdot\left\{\left[\mathbf{X}^{\prime}\left(z_{d}\right)+\frac{E I}{\kappa G A} \mathbf{X}^{\prime \prime \prime}\left(z_{d}\right)\right]^{T}\right. \\
& \left.\cdot\left[\mathbf{X}^{\prime}\left(z_{d}\right)+\frac{E I}{\kappa G A} \mathbf{X}^{\prime \prime \prime}\left(z_{d}\right)\right]\right\} \dot{\boldsymbol{\eta}}-2 \dot{\mathbf{q}}_{\mathbf{u}}^{\mathrm{T}} \dot{\psi} \Psi^{\mathrm{T}} \mathbf{V q}_{\mathbf{v}} \\
& +2 \dot{\mathbf{q}}_{\mathbf{v}}^{\mathrm{T}} \dot{\psi} \mathbf{V}^{\mathrm{T}} \boldsymbol{\Psi} \mathbf{q}_{\mathbf{u}}-2 \dot{\boldsymbol{\eta}}^{\mathrm{T}} \dot{\psi} \mathbf{X}^{\mathrm{T}}\left(z_{d}\right) \boldsymbol{\Psi} \sin \psi \mathbf{q}_{\mathbf{u}} \\
& -2 \dot{\boldsymbol{\eta}}^{\mathrm{T}} \dot{\psi} \mathbf{X}^{\mathrm{T}}\left(z_{d}\right) \mathbf{V} \cos \psi \mathbf{q}_{\mathbf{v}}+2 \dot{\boldsymbol{\xi}}^{\mathrm{T}} \dot{\psi} \mathbf{Y}^{\mathrm{T}}\left(z_{d}\right) \boldsymbol{\Psi} \cos \psi \mathbf{q}_{\mathbf{u}} \\
& -2 \dot{\xi}^{\mathrm{T}} \dot{\psi} \mathbf{Y}^{\mathrm{T}}\left(z_{d}\right) \mathbf{V} \sin \psi \mathbf{q}_{\mathbf{v}}-2 \dot{\boldsymbol{\eta}}^{\mathrm{T}} \dot{\psi}(R+x) \mathbf{X}^{\mathrm{T}} \boldsymbol{\Phi} \\
& \cdot \cos \psi \mathbf{q}_{\boldsymbol{\theta}}-2 \dot{\boldsymbol{\xi}}^{\mathrm{T}} \dot{\psi}(R+x) \mathbf{Y}^{\mathrm{T}}\left(z_{d}\right) \boldsymbol{\Phi} \sin \psi \mathbf{q}_{\boldsymbol{\theta}} \\
& +4 \dot{\mathbf{q}}_{\boldsymbol{\theta}}^{\mathrm{T}} \dot{\psi}(R+x) \boldsymbol{\Phi}^{\mathrm{T}} \boldsymbol{\Psi} \mathbf{q}_{\mathbf{u}}+2 \dot{\boldsymbol{\xi}}^{\mathrm{T}} \mathbf{Y}^{\mathrm{T}}\left(z_{d}\right) \mathbf{V} \cos \psi \dot{\mathbf{q}}_{\mathbf{v}} \\
& U_{b}=\frac{1}{2} E_{b} A_{b} \int_{0}^{L_{b}} \mathbf{q}_{u}^{\mathrm{T}} \boldsymbol{\Psi}^{\prime \mathrm{T}} \boldsymbol{\Psi}^{\prime} \mathbf{q}_{\mathbf{u}} \mathrm{d} x+\frac{1}{2} \\
& \cdot E_{b} I_{b} \int_{0}^{L_{b}} \mathbf{q}_{\mathbf{v}}^{\mathrm{T}} \mathbf{V}^{\prime \prime \mathbf{T}} \mathbf{V}^{\prime \prime} \mathbf{q}_{\mathbf{v}} \mathrm{d} x+\frac{1}{4} \\
& \cdot \mathbf{q}_{\mathbf{v}}^{\mathbf{T}} \rho_{b} A_{b} \dot{\psi}^{2} \int_{0}^{L_{b}}\left[L_{b}^{2}-x^{2}+2 R\left(L_{b}-x\right)\right] \\
& \cdot \mathbf{V}^{\prime T} \mathbf{V}^{\prime} \mathrm{d} x \mathbf{q}_{\mathbf{v}} \text {. }
\end{aligned}
$$

Substituting kinetic energy equation (32) and potential energy equation (30) into simplified Lagrange equation, the differential equation of vibration of $i$ th blade is obtained as follows:

$$
\mathbf{M} \ddot{\mathbf{q}}+(\mathbf{C}+\mathbf{G}) \dot{\mathbf{q}}+\mathbf{K q}=0
$$

where $\mathbf{M}, \mathbf{C}, \mathbf{G}$, and $\mathbf{K}$ are mass, damping, gyroscopic, and stiffness matrices of the blade.

2.3. Matrices Assembly of the Rotor-Blade Coupling System. Discretize the differential equations of motion of the $i$ th blade and the rotor system obtained and write in matrix forms. Because each blade has a corresponding mass matrix $M$, damping matrix $C$, gyroscopic matrix $G$, and stiffness matrix 


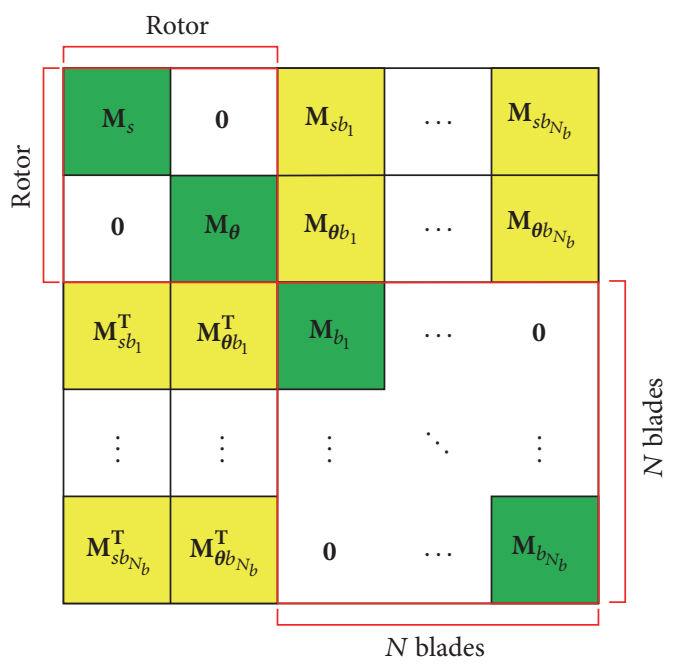

(a) Mass matrix

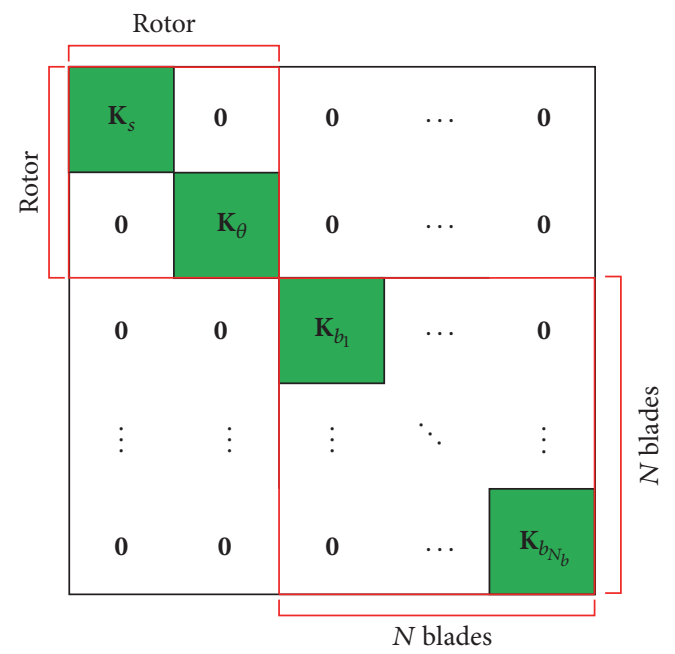

(c) Stiffness matrix

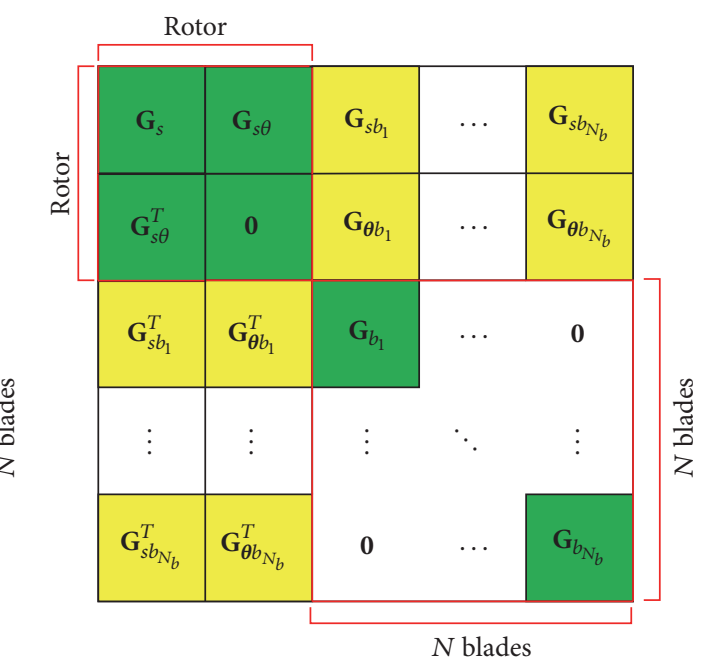

(b) Gyroscopic matrix

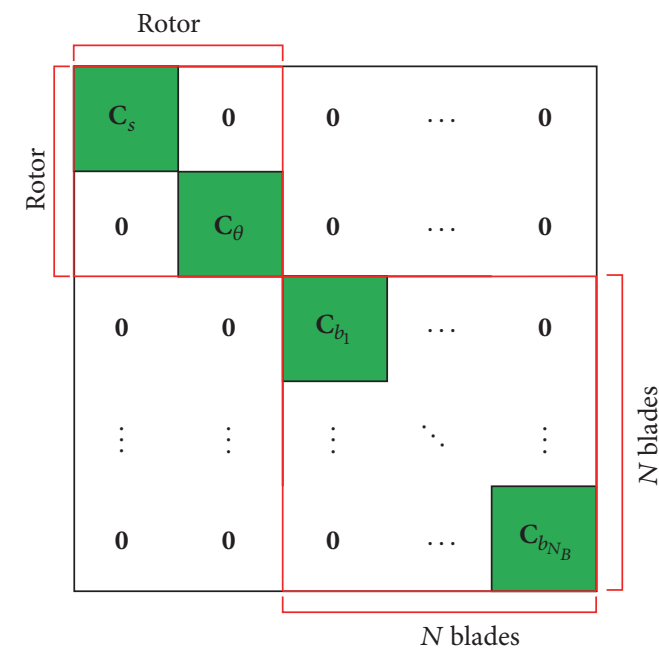

(d) Damping matrix

FIGURE 4: Schematic diagram of assembly of matrices of the rotor-blade coupling system.

$K$ and blade-disk system is cyclic symmetric structure, the matrices of each blade and rotor system can be assembled to the global matrix of rotor-blade coupling system. The mass, gyroscopic, stiffness, and damping matrices have been assembled as shown in Figure 4.

Assembled differential equations of motion of a rotorblade coupled system are written as

$$
\mathbf{M}_{r b} \ddot{\mathbf{q}}_{r b}+\mathbf{C}_{r b} \dot{\mathbf{q}}_{r b}+\mathbf{K}_{r b} \mathbf{q}_{r b}=0,
$$

where the meaning of the parameters in the formula is shown in Appendix C.

\section{Dynamic Eigenvalue Analysis of a Rotor-Blade Coupling System}

In order to further study the vibration characteristics of the coupling system, it is necessary to analyze the critical speed. Specific parameters are shown in "Geometric and Material Properties of Rotor-Blade System."
In order to reduce the length of article, only these effects on critical speed of rotor-blade coupling system are studied in this section: the number of blades $N_{b}$ from three to seven, the position of disk, and the support stiffness of shaft.

\subsection{The Influence of Blades Number on Critical Speed}

3.1.1. When the Rotational Speed $\omega=0$. Figure 5 shows how the first two-order frequencies of blade change due to the number of blades in a three-to-seven-blade rotor when the position of disk is different. $1 a_{1}$ and $1 b_{1}$ belong to a set where the blade's first mode predominates in the rotorblade coupling system. $1 c_{1}$ and $1 d_{1}$ belong to a set where the blade's second mode predominates in the shaft-blade coupling system. Note that there are two reference marks at 163.15 and 1022.5 , respectively, denoting the cantilevered blade's first- and second-order frequency. Because of the coupling between shaft and blade, there are two coupling modes dominated by blade's first two-order modes which 


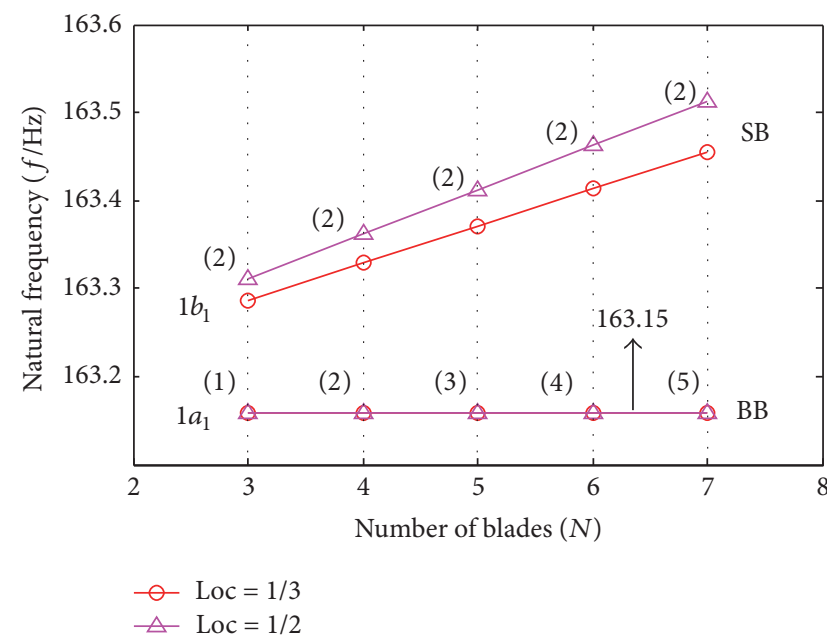

(a) First-order frequency changing diagram

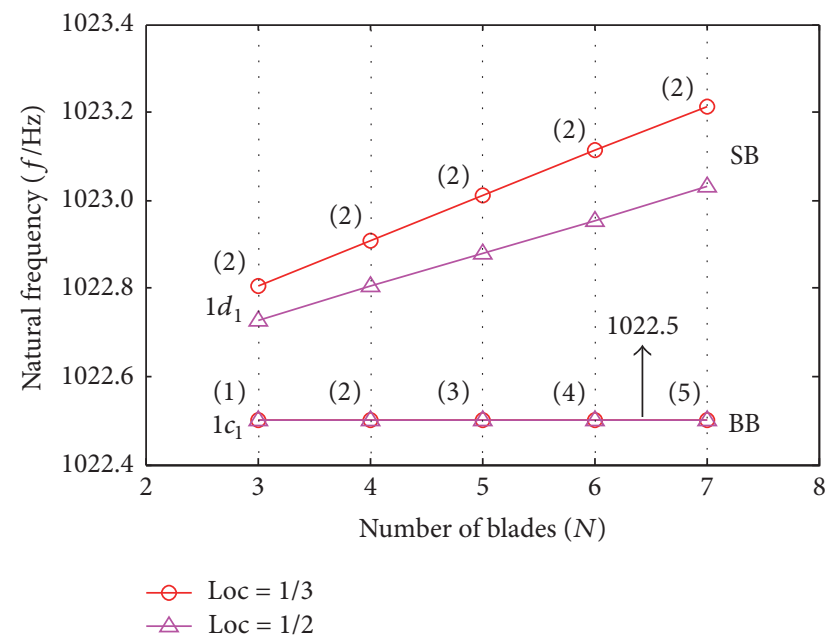

(b) Second-order frequency changing diagram

Figure 5: Frequency of blade changes due to the number of blades in a three-to-seven-blade rotor.

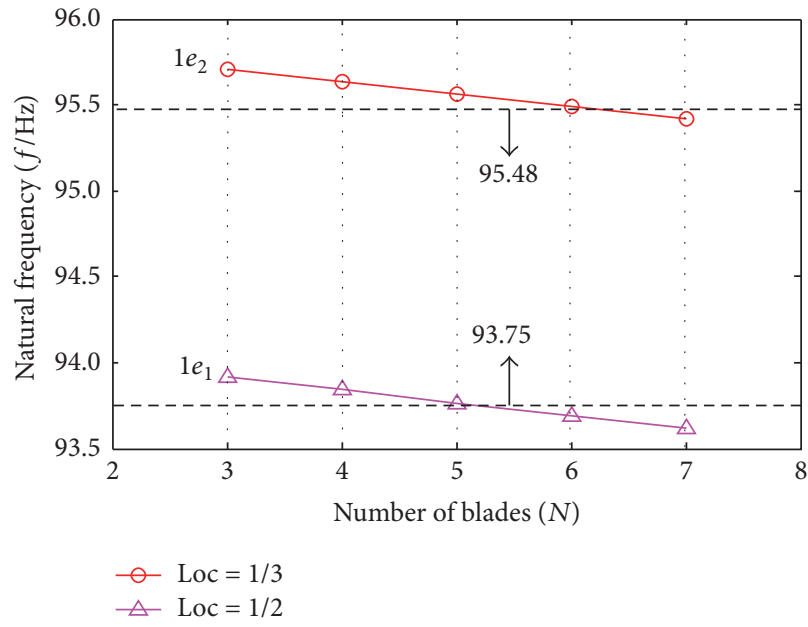

(a) First-order frequency changing diagram

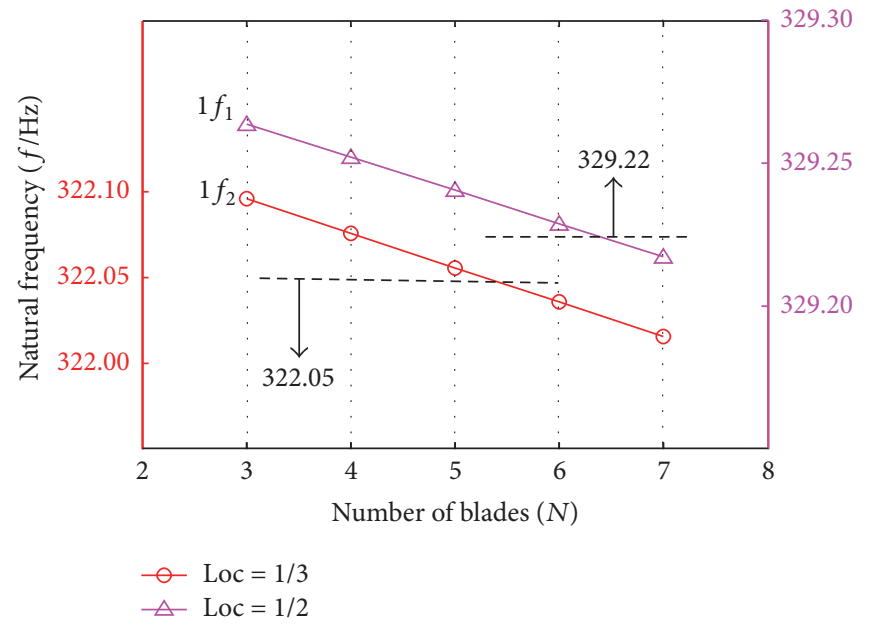

(b) Second-order frequency changing diagram

FIGURE 6: Frequency of rotor changes due to the number of blades in a three-to-seven-blade rotor.

could be found: shaft-blade (SB) and blade-blade (BB). What is more, it also reveals a significant phenomenon that the $\mathrm{BB}$ modes are of repeated frequencies of $\left(N_{b}-2\right)$ multiplicity and the SB modes are of repeated frequencies of (2) multiplicity as two transverse vibration directions of shaft are taken into consideration. And with the increase of the number of blades, the frequency of SB mode increases linearly but that of BB mode is constant. In addition, it also shows frequency changes of both two modes, respectively, when the disk is located in $1 / 3$ and $1 / 2$ of the shaft. It could be found that the frequency of $\mathrm{BB}$ mode does not change but the frequency of SB mode changes with the change of disk position, and the changing trend of blades number between first- and secondorder modes is different.

Figure 6 demonstrates how the first two-order frequencies of rotor change due to the number of blades in a three-to-seven-blade rotor when the position of disk is different. It could be found that $1 e_{1}$ and $1 e_{2}$ belong to a set where the rotor's first mode predominates in the shaftblade coupling system and $1 f_{1}$ and $1 f_{2}$ belong to a set where the rotor's second mode predominates in the shaft-blade coupling system. Note that $93.75 \mathrm{~Hz}, 95.48 \mathrm{~Hz}, 322 \mathrm{~Hz}$, and $329.22 \mathrm{~Hz}$, respectively, denoting the rotor's first- and secondorder frequency when the disk is located in $1 / 3$ and $1 / 2$ of the shaft. Furthermore, it also shows that the frequency of rotor decreases with the increase of the number of blades and the trend of decrease is mainly linear. In addition, it is also found that the position of disk can also have a certain influence on the vibration of shaft-blade coupling system. Therefore, it will be investigated in next section.

3.1.2. When the Rotational Speed $\omega \neq 0$. Figure 7 illustrates how Campbell diagram of blade changes due to the number of blades. $1 a_{1}, 1 b_{1}$, and $1 b_{2}$ belong to a set where the blade's 


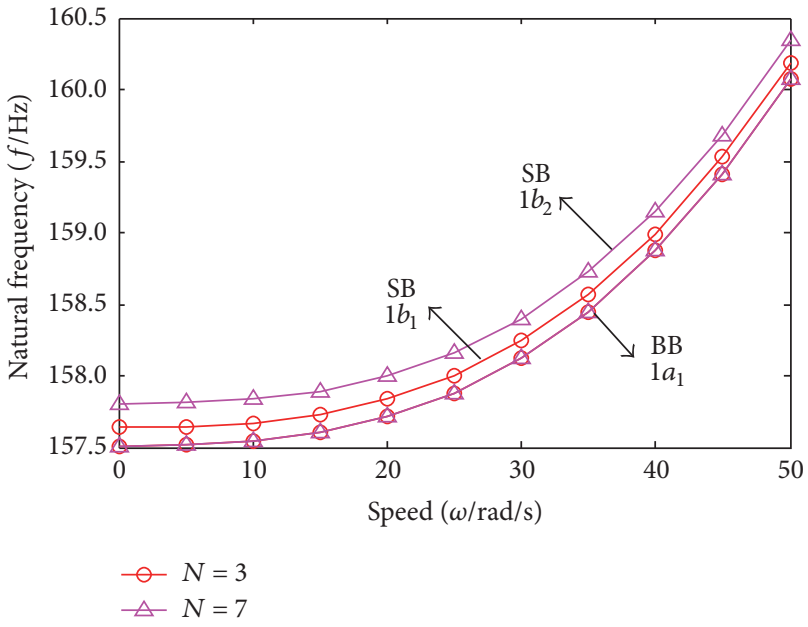

(a) First-order Campbell diagram

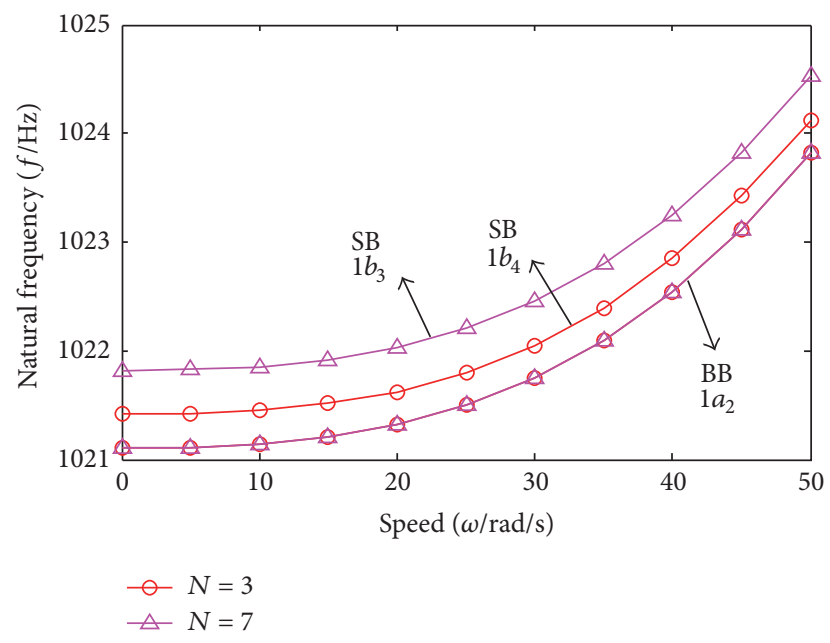

(b) Second-order Campbell diagram

Figure 7: Campbell diagram of blade changes due to the number of blades.

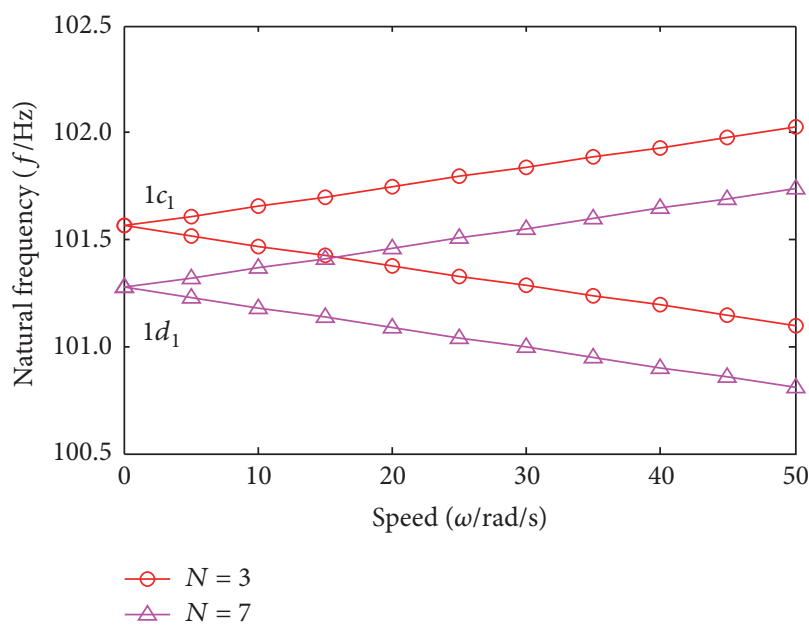

(a) First-order Campbell diagram

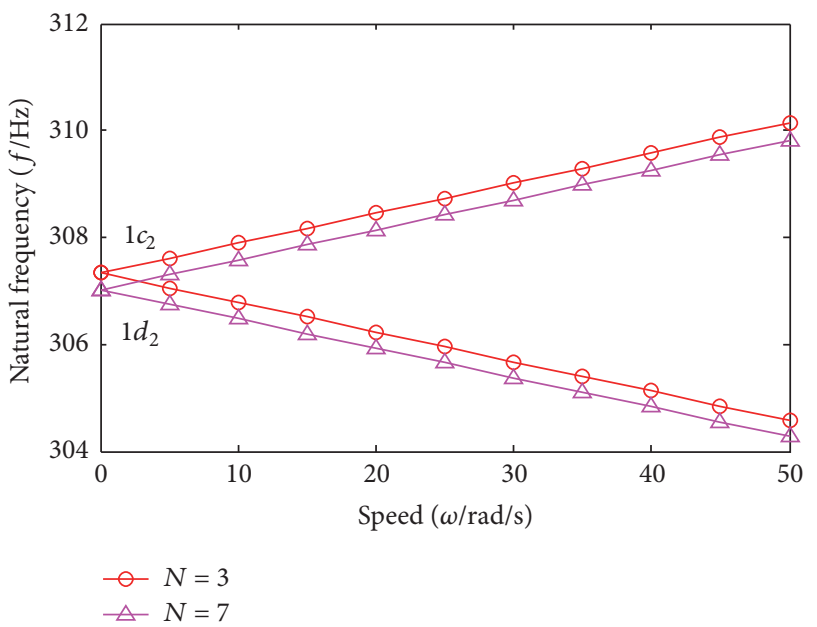

(b) Second-order Campbell diagram

Figure 8: Campbell diagram of rotor changes due to the number of blades.

first mode predominates in the rotor-blade coupling system. $1 a_{2}, 1 b_{3}$, and $1 b_{4}$ belong to a set where the blade's second mode predominates in the rotor-blade coupling system. It could be found that the Campbell diagram of blade is affected by the number of blades, and with the increase of the number of blades, frequency and corresponding Campbell diagram of BB mode are constant, but frequency of SB mode increases and corresponding Campbell diagram of SB mode shows the trend of increasing. These phenomena can be verified with Figure 5.

Campbell diagram of rotor changes due to the number of blades are shown in Figure $8.1 c_{1}$ and $1 d_{1}$ belong to a set where the rotor's first mode predominates in the rotorblade coupling system and $1 c_{2}$ and $1 d_{2}$ belong to a set where the rotor's second mode predominates in the rotor-blade coupling system. It could be found that the Campbell diagram of rotor is affected by the number of blades, and with the increase of the number of blades, frequency of rotor decreases and corresponding Campbell diagram of it shows the trend of decreasing. What is more, the trend is more obvious in the first-order Campbell diagram. These phenomena can be verified with Figure 6.

\subsection{The Influence of Disk Position on Critical Speed}

3.2.1. When the Rotational Speed $\omega=0$. Figure 9 shows how the first two-order frequencies of blade change due to the position of disk when the number of blades is different. $1 a_{1}$, $1 b_{1}$, and $1 b_{2}$ belong to a set where the blade's first mode predominates in the rotor-blade coupling system. $1 a_{2}, 1 b_{3}$, and $1 b_{4}$ belong to a set where the blade's second mode predominates in the rotor-blade coupling system. Note that 


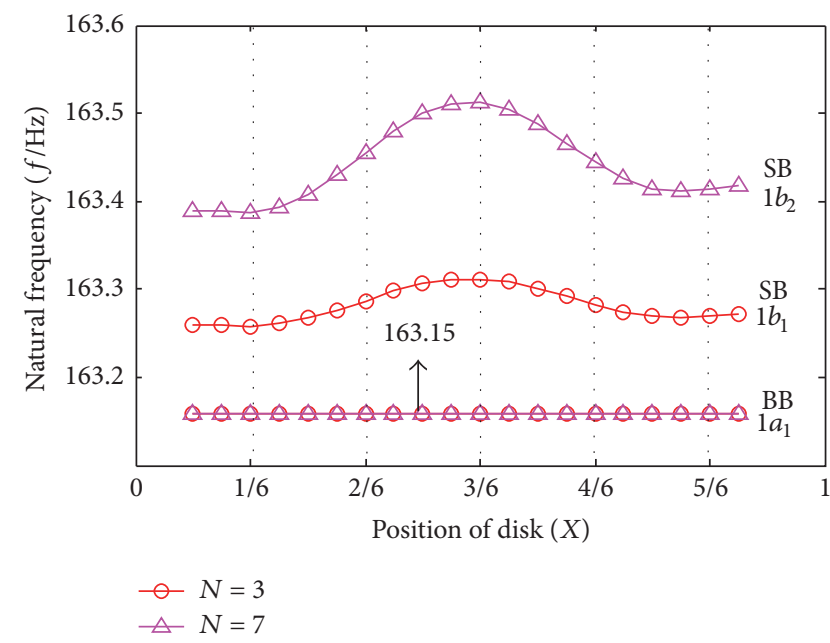

(a) First-order frequency changing diagram

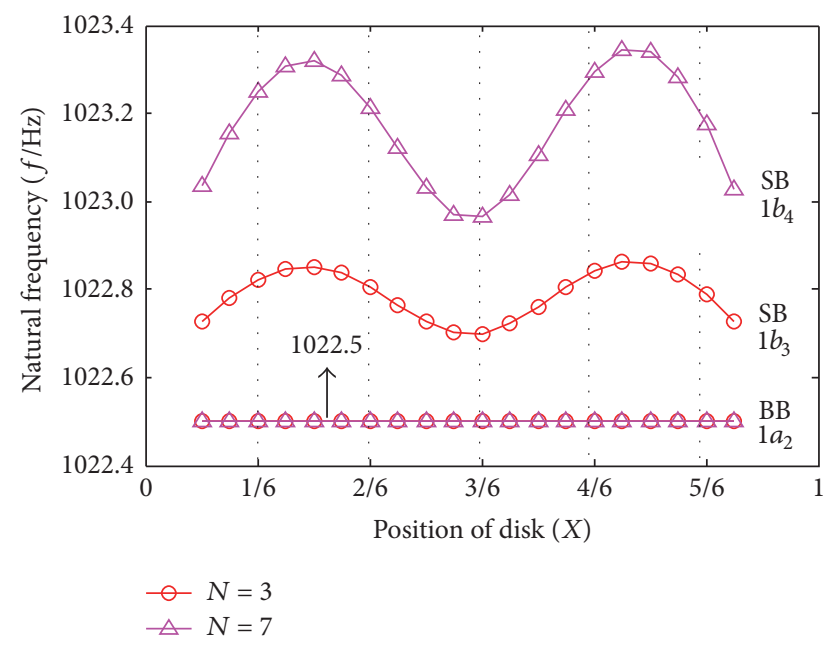

(b) Second-order frequency changing diagram

FIgURE 9: Frequency of blade changes due to the position of disk.

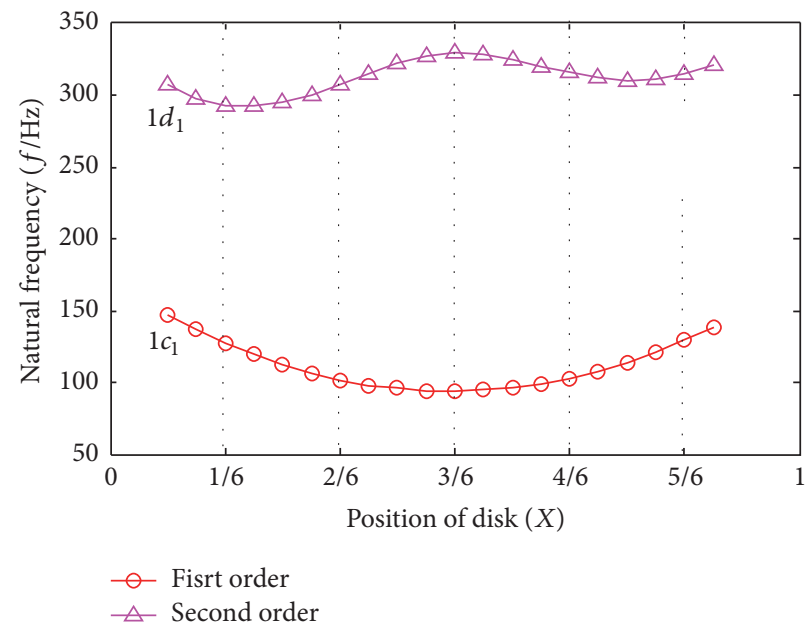

(a) 3-blade rotor-blade coupling system changing diagram

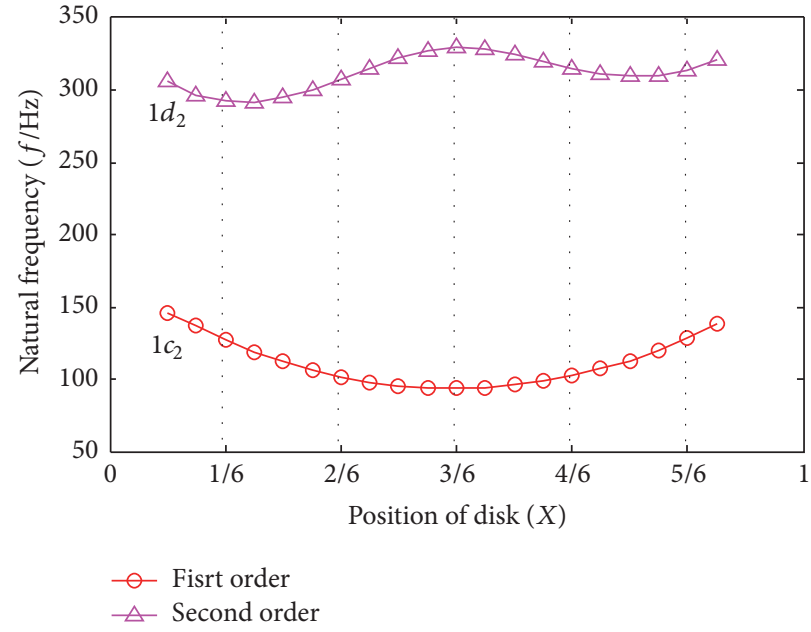

(b) 7-blade rotor-blade coupling system changing diagram

FIGURE 10: Frequency of rotor changes due to the position of disk.

there are two reference marks at $163.15 \mathrm{~Hz}$ and $1022.5 \mathrm{~Hz}$, respectively, denoting the cantilevered blade's first- and second-order frequency. It could be found that the natural frequency of the SB mode is affected by the position of the disk. And the influence is symmetrical about the middle of shaft. When the disk moves from the end to the center of the shaft, the first-order frequency increases gradually and reaches the maximum when the disk is in the center of shaft, but the second-order frequency increases first and then decreases and reaches the minimum when the disk is in the center of shaft. What is more, the trend of the change is more obvious with the increase of the number of blades.

Figure 10 illustrates how first two-order frequencies of rotor change due to the position of disk in a three-to-sevenblade rotor when the number of blades is different. $1 c_{1}$ and $1 c_{2}$, respectively, belong to a set where the rotor's first mode predominates in the three-blade and seven-blade rotor-blade coupling system. $1 d_{1}$ and $1 d_{2}$, respectively, belong to a set where the rotor's second mode predominates in the threeblade and seven-blade rotor-blade coupling system. It could be found that the natural frequency of rotor is affected by the position of the disk and changes symmetrically with the center of shaft. When the disk moves from the end to the center of the shaft, the first-order frequency decreases gradually and reaches the minimum when the disk is in the center of shaft, but the second-order frequency decreases first and then increases and reaches the maximum when the disk is in the center of shaft. What is more, the trend of change is not affected by the number of blades.

3.2.2. When the Rotational Speed $\omega \neq 0$. Figure 11 demonstrates how the Campbell diagram of blade changes due 


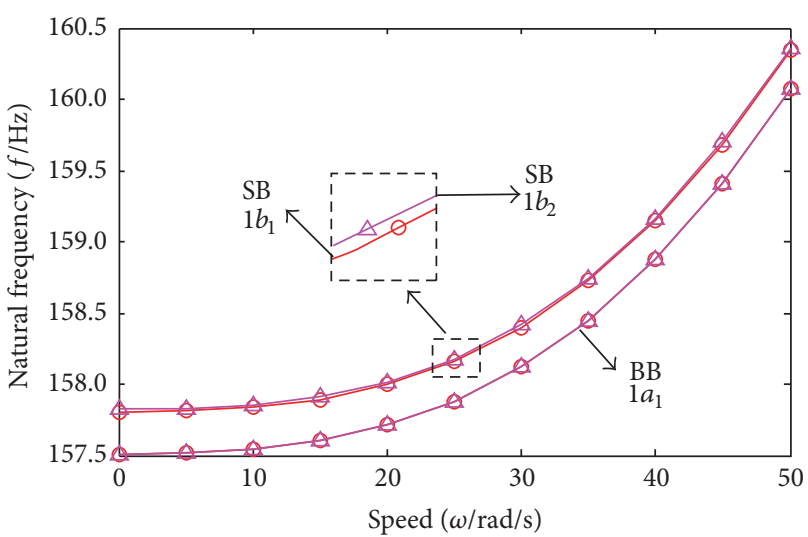

$\bigcirc \operatorname{Loc}=1 / 3$

$\triangle \operatorname{Loc}=1 / 2$

(a) First-order Campbell diagram

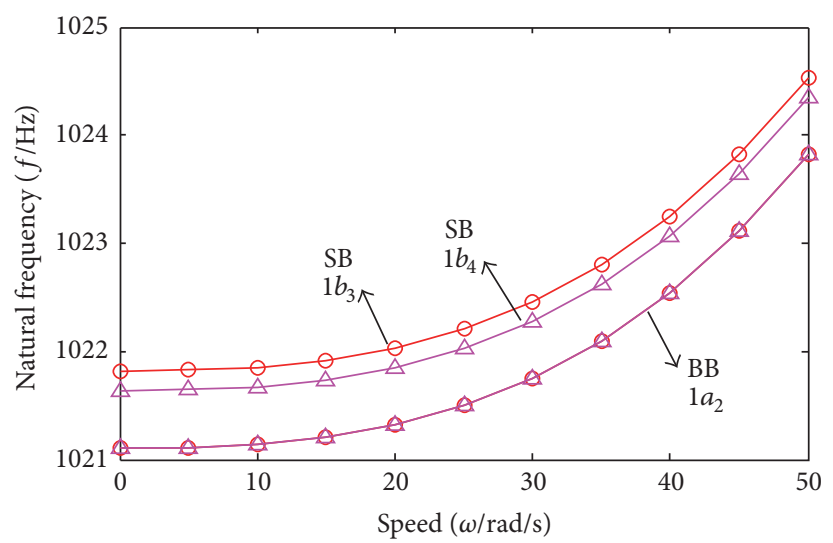

$\multimap \operatorname{Loc}=1 / 3$

$\triangle \operatorname{Loc}=1 / 2$

(b) Second-order Campbell diagram

FIGURE 11: Campbell diagram of blade changes due to the position of disk.

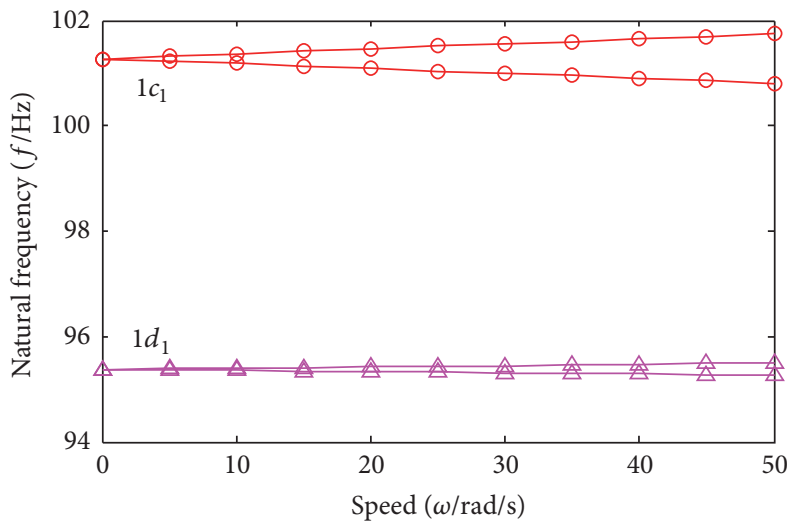

- $\operatorname{Loc}=1 / 3$

$\triangle \operatorname{Loc}=1 / 2$

(a) First-order Campbell diagram

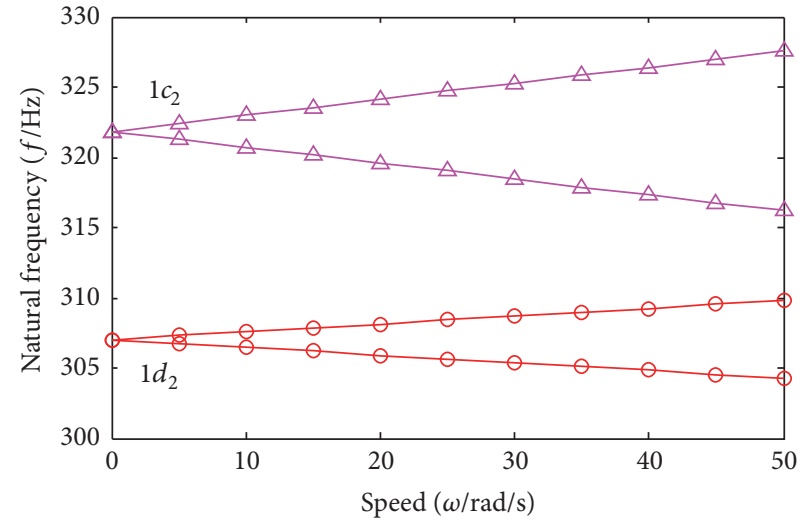

$\bigcirc \operatorname{Loc}=1 / 3$

$\triangle \operatorname{Loc}=1 / 2$

(b) Second-order Campbell diagram

Figure 12: Campbell diagram of rotor changes due to the position of disk.

to the position of disk. $1 a_{1}, 1 b_{1}$, and $1 b_{2}$ belong to a set where the blade's first mode predominates in the rotorblade coupling system. $1 a_{2}, 1 b_{3}$, and $1 b_{4}$ belong to a set where the blade's second mode predominates in the rotorblade coupling system. It could be found that the Campbell diagram of blade is affected by the position of disk. When the disk moves from $1 / 3$ to the center of the shaft, firstorder frequency and corresponding Campbell diagram of $\mathrm{BB}$ mode are constant, and first-order frequency of SB mode increases and corresponding Campbell diagram of SB mode shows the trend of increasing but second-order frequency and corresponding Campbell diagram behave oppositely. These phenomena can be verified with Figure 9.

Campbell diagram of rotor changes due to the position of disk is shown in Figure 12. $1 c_{1}$ and $1 d_{1}$ belong to a set where the rotor's first mode predominates in the rotorblade coupling system. $1 c_{2}$ and $1 d_{2}$ belong to a set where the rotor's second mode predominates in the rotor-blade coupling system. It could be found that the Campbell diagram of rotor is affected by the position of disk. When the disk moves from $1 / 3$ to the center of the shaft, firstorder frequency of rotor decreases and corresponding Campbell diagram of rotor shows the trend of decreasing but second-order frequency and corresponding Campbell diagram behaves oppositely. These phenomena can be verified with Figure 10.

3.3. The Influence of Support Stiffness on Critical Speed. In order to make the effect of support stiffness on critical speed more obvious, in this section $N_{b}=7, \mathrm{D} \_$loc $=1 / 2$. 


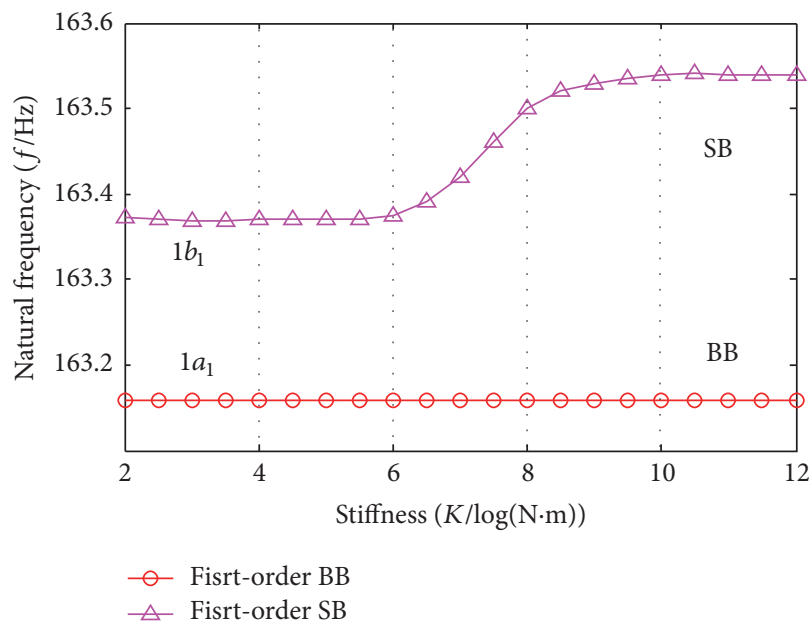

(a) First-order frequency changing diagram

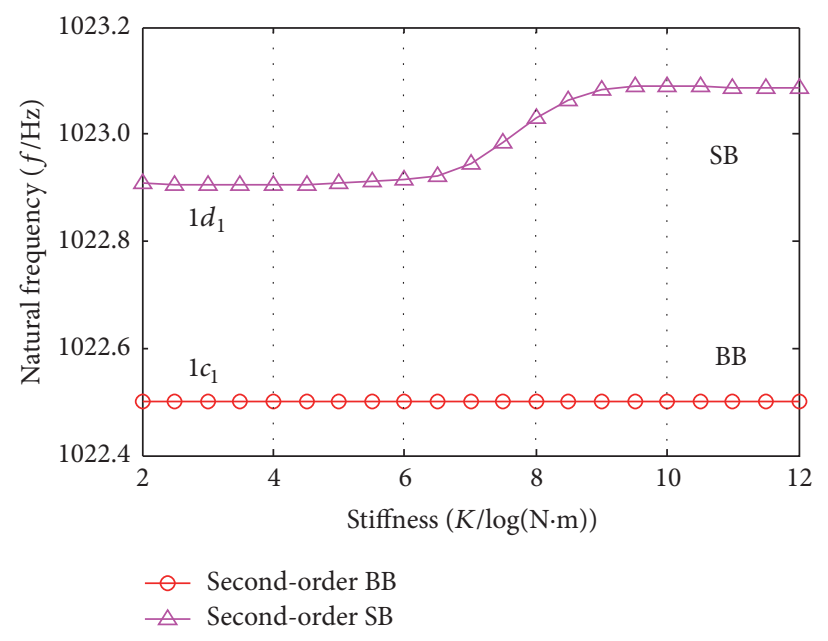

(b) Second-order frequency changing diagram

FIGURE 13: Frequency of blade changes due to the support stiffness of shaft.

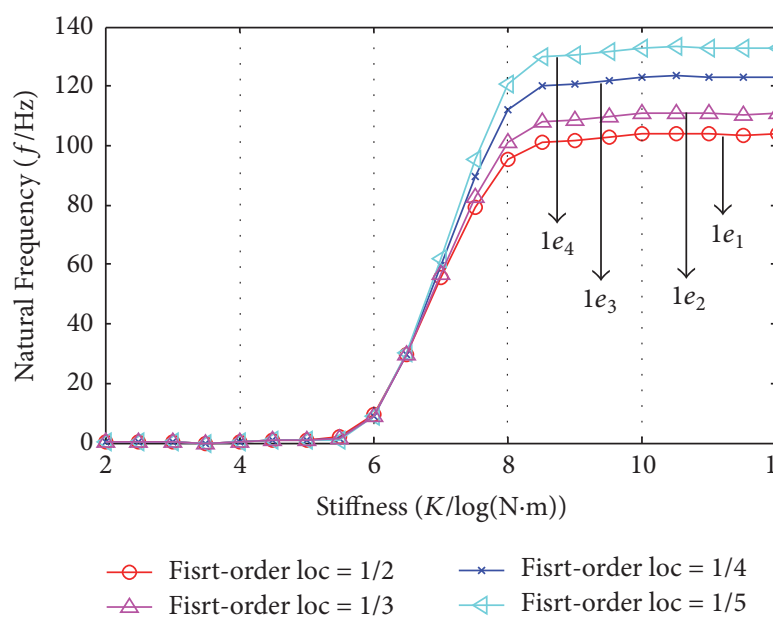

(a) First-order frequency changing diagram

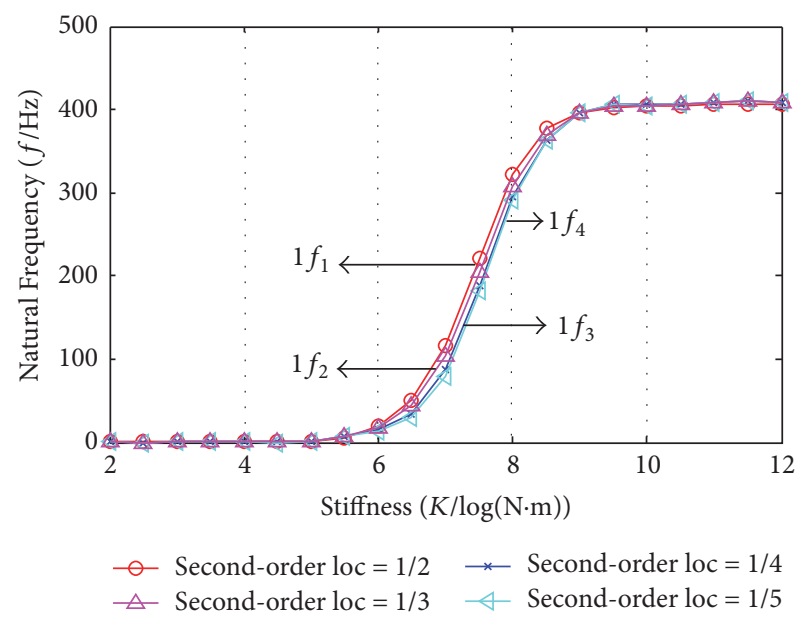

(b) Second-order frequency changing diagram

FIGURE 14: Frequency of rotor changes due to the support stiffness of shaft.

3.3.1. When the Rotational Speed $\omega=0$. Figure 13 shows how the first two-order frequencies of blade change due to the support stiffness of shaft. $1 a_{1}$ and $1 b_{1}$ belong to a set where the blade's first mode predominates in the rotor-blade coupling system. $1 a_{2}, 1 c_{1}$, and $1 d_{1}$ belong to a set where the blade's second mode predominates in the rotor-blade coupling system. It could be found that the natural frequency of $\mathrm{BB}$ mode is constant but that of SB mode is constant at first and then increases with the increase of support stiffness of shaft. What is more, the increasing trend of first-order frequency is more obvious. That is, because the first-order frequency of blade is closer to that of shaft, the coupling phenomenon is more obvious.

Figure 14 demonstrates how the first two-order frequencies of rotor change due to the support stiffness of shaft. $1 e_{1}$, $1 e_{2}, 1 e_{3}$, and $1 e_{4}$ belong to a set where the rotor's first mode predominates in the rotor-blade coupling system. $1 f_{1}, 1 f_{2}$,
$1 f_{3}$, and $1 f_{4}$ belong to a set where the blade's second mode predominates in the rotor-blade coupling system. It could be found that the natural frequency of rotor is affected by the support stiffness quite obviously. When the support stiffness is small, the frequency of rotor is nearly zero. However, when the support stiffness reaches a critical point, the frequency of rotor increases rapidly with the increase of support stiffness. When the support stiffness is large enough, the frequency of rotor is constant.

3.3.2. When the Rotational Speed $\omega \neq 0$. Figure 15 illustrates how Campbell diagram of blade changes due to the support stiffness of shaft. $1 a_{1}, 1 b_{1}, 1 b_{2}$, and $1 b_{3}$ belong to a set where the blade's first mode predominates in the rotor-blade coupling system. $1 a_{2}, 1 b_{4}, 1 b_{5}$, and $1 b_{6}$ belong to a set where the blade's second mode predominates in the rotor-blade coupling system. It could be found that, with the increase of 


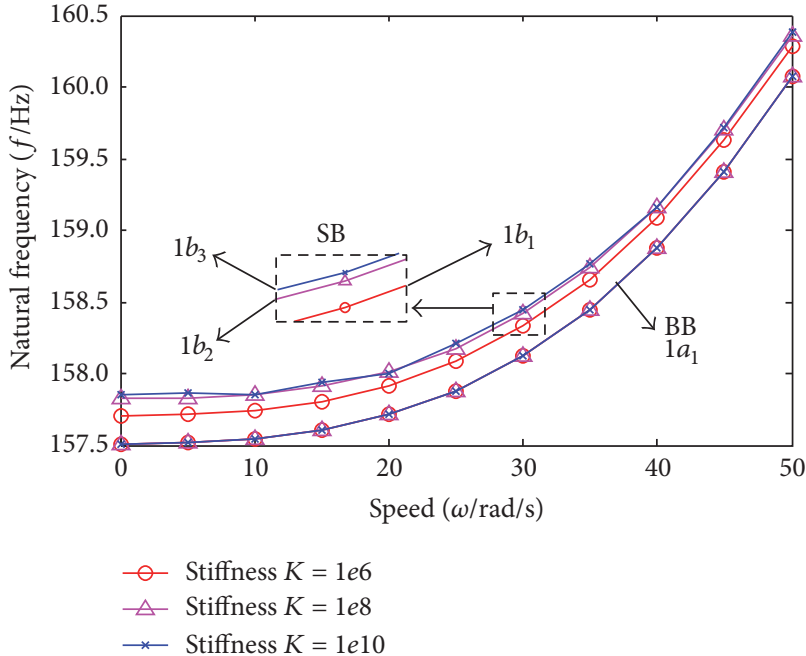

(a) First-order Campbell diagram

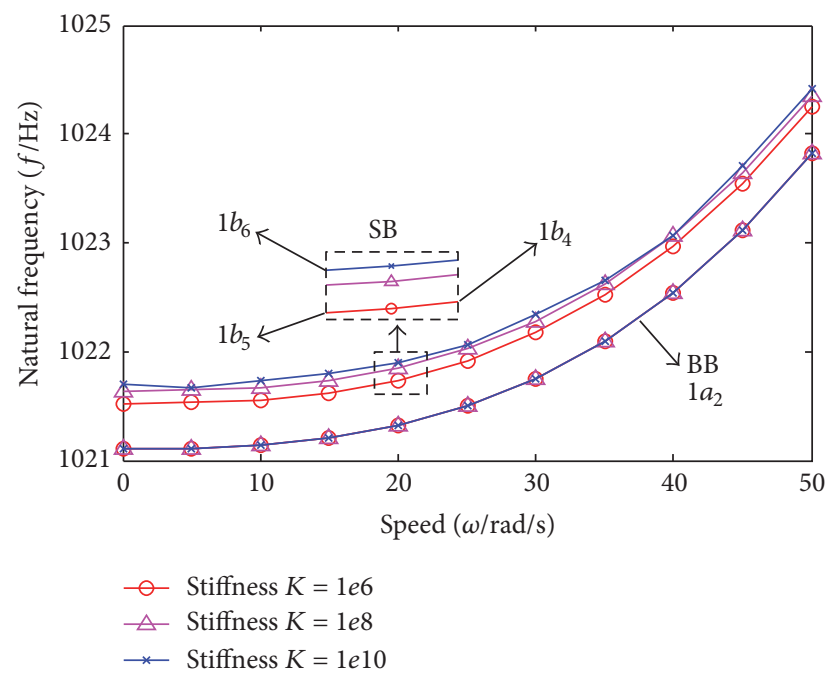

(b) Second-order Campbell diagram

FIGURE 15: Campbell diagram of blade changes due to support stiffness of shaft.

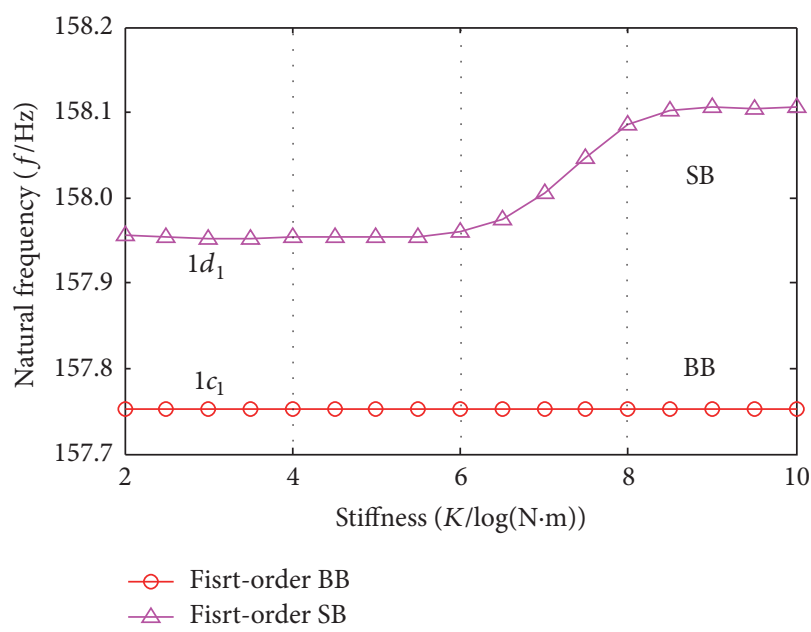

(a) First-order critical speed changing diagram

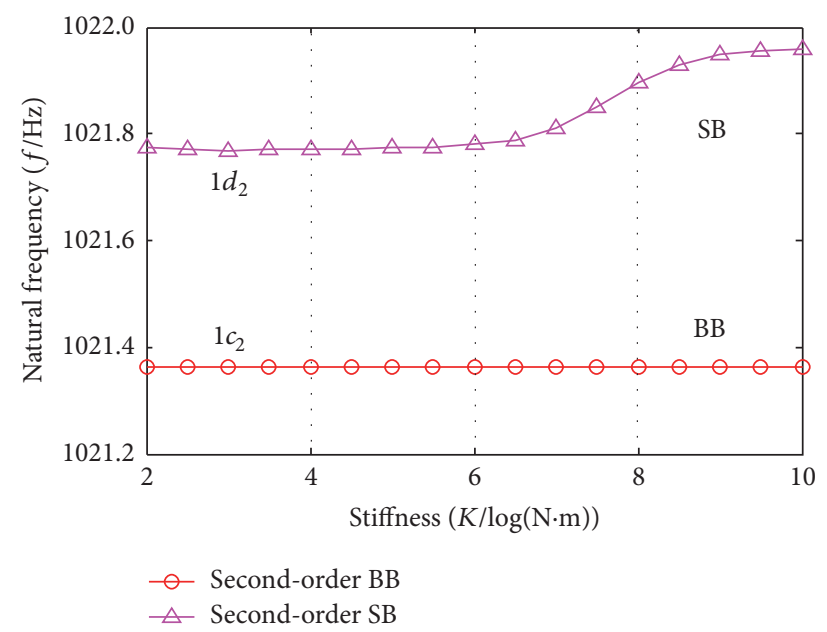

(b) Second-order critical speed changing diagram

FIGURE 16: Critical speed of blade changes due to the support stiffness of shaft.

support stiffness, the frequency and corresponding Campbell diagram of $\mathrm{BB}$ mode are constant, but the frequency of SB mode increases and corresponding Campbell diagram of SB mode shows the trend of increasing. These phenomena can be verified with Figure 9.

In order to further analyze the influence of the change of support stiffness, it is analyzed that the critical speed of blade changes due to the support stiffness of shaft when $\omega=$ $30 \mathrm{rad} / \mathrm{s}$.

First two-order critical speeds of blade change due to the support stiffness of shaft are shown in Figure 16. $1 c_{1}$ and $1 d_{1}$ belong to a set where the blade's first mode predominates in the rotor-blade coupling system. $1 a_{2}, 1 c_{2}$, and $1 d_{2}$ belong to a set where the blade's second mode predominates in the rotor-blade coupling system. It could be found that the critical speed is affected by support stiffness. The critical speed of BB mode is constant but that of SB mode is constant at first and then increases with the increase of support stiffness of shaft. What is more, the trend of increasing of first-order frequency is more obvious.

Figure 17 shows how the Campbell diagrams of rotor change due to the support stiffness of shaft. $1 e_{1}, 1 f_{1}$, and $1 g_{1}$ belong to a set where the rotor's first mode predominates in the rotor-blade coupling system. $1 e_{2}, 1 f_{2}$, and $1 g_{2}$ belong to a set where the blade's second mode predominates in the rotor-blade coupling system. It could be found that the Campbell diagram of rotor is affected by support stiffness and with the increase of support stiffness, the natural frequency 


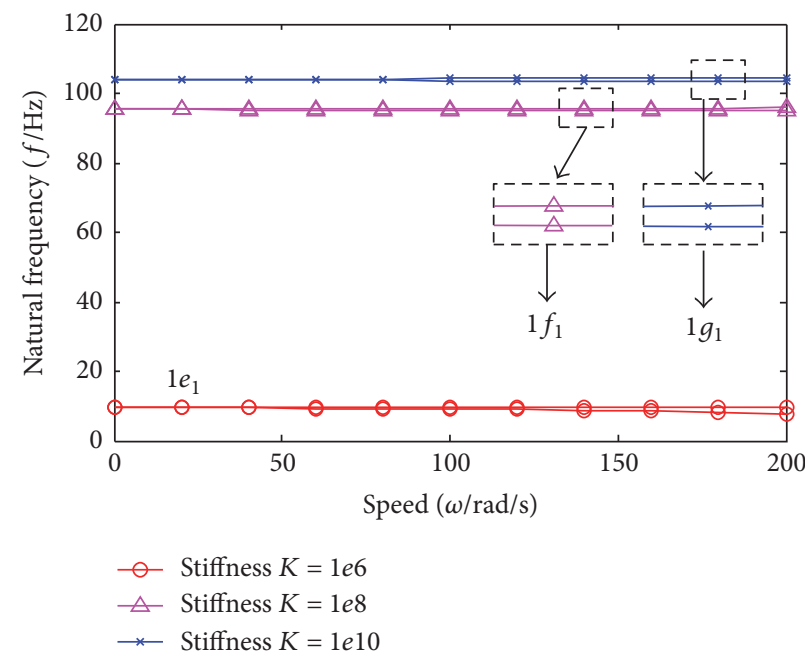

(a) First-order Campbell diagram

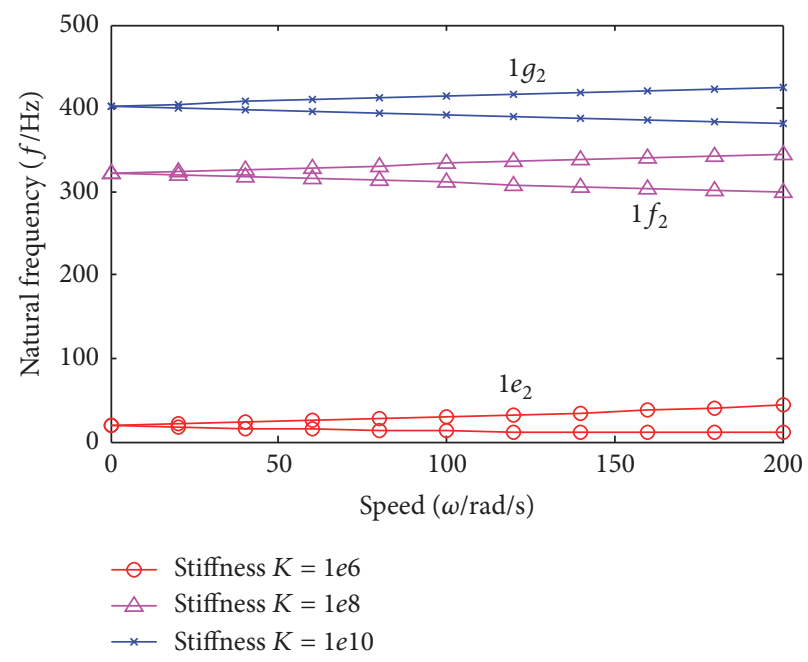

(b) Second-order Campbell diagram

FIGURE 17: Campbell diagram of rotor changes due to support stiffness of shaft.

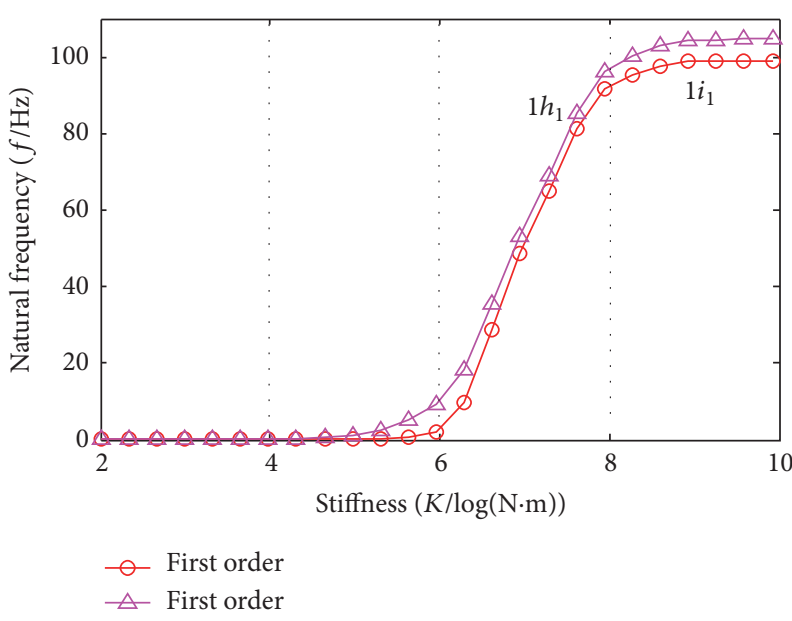

(a) First-order critical speed changing diagram

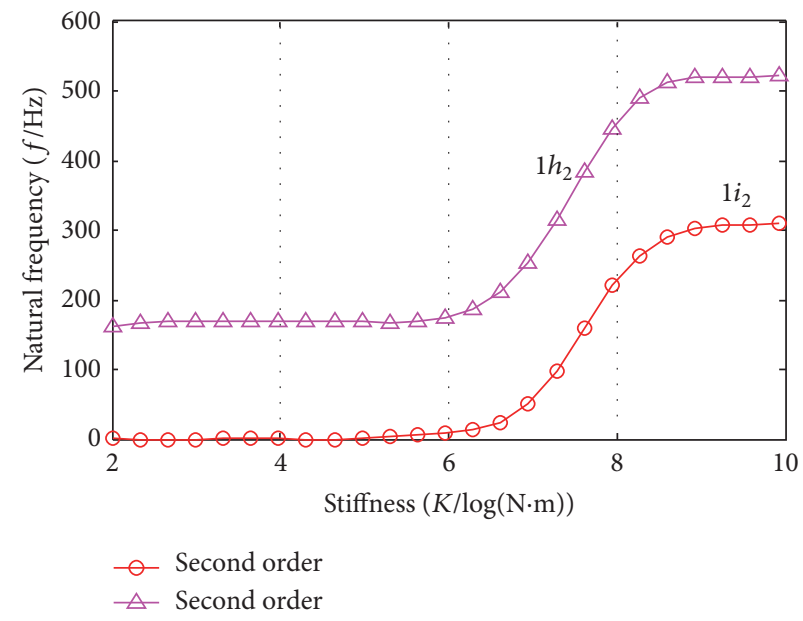

(b) Second-order critical speed changing diagram

FIGURE 18: Critical speed of rotor changes due to the support stiffness of shaft.

of rotor increases and corresponding Campbell diagram of rotor shows the trend of increasing. These phenomena can be verified with Figure 10.

In order to further analyze the influence of the change of support stiffness and make the change more obvious, it is analyzed that the critical speed of rotor changes due to the support stiffness of shaft when $\omega=1000 \mathrm{rad} / \mathrm{s}$.

Figure 18 demonstrates how the first two-order critical speeds of rotor change due to the support stiffness of shaft. $1 h_{1}$ and $1 i_{1}$ belong to a set where the rotor's first mode predominates in the rotor-blade coupling system. $1 h_{2}$ and $1 i_{2}$ belong to a set where the rotor's second mode predominates in the rotor-blade coupling system. It could be found that the critical speed is affected by support stiffness. The critical speed of rotor increases with the increase of support stiffness of shaft. What is more, the trend of increasing of second-order frequency is more obvious.

\section{Analysis of Mode Shapes}

In order to further analyze the vibration characteristics of rotor-blade coupling system and verify the above phenomena, the influence of different factors on mode shapes of system is analyzed in detail. In order to make the analysis more reasonable, the mode shape of first-order natural frequency of the blade is analyzed prior to the formal analysis. Here, the number of blades $N_{b}=4$ and the support stiffness of shaft $K=1 \times 10^{7} \mathrm{~N} / \mathrm{m}$.

Figure 19 demonstrates the mode shape of 4-blade rotorblade coupling system. It could be found that there is no 


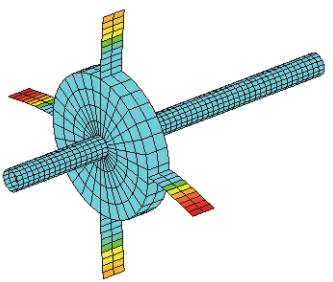

(a) SB mode of blade

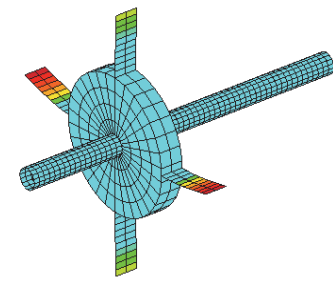

(b) BB mode of blade

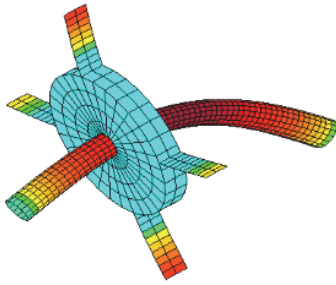

(c) First-order mode of rotor

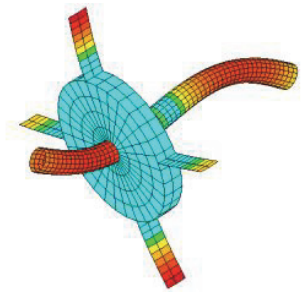

(d) Second-order mode of rotor

FIGURE 19: Mode shape of 4-blade rotor-blade coupling system.

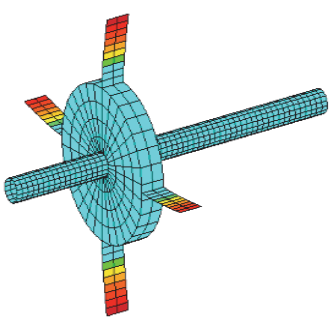

(a) Mode of blade (4 blades)

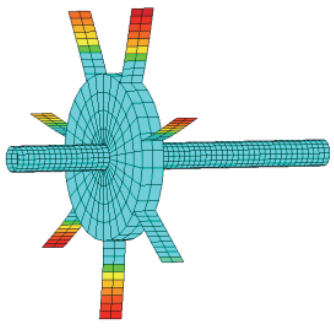

(b) Mode of blade (7 blades)

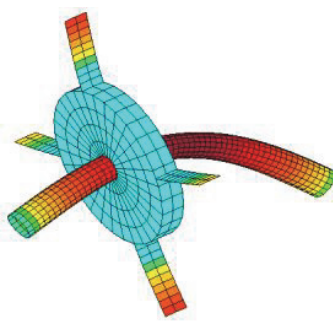

(c) Mode of rotor (4 blades)

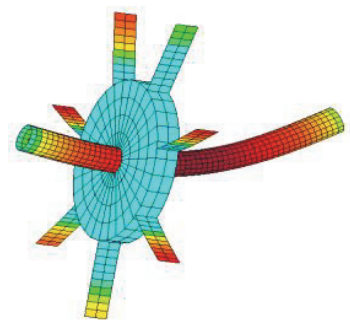

(d) Mode of rotor (7 blades)

Figure 20: Mode shapes of rotor-blade coupling system change due to the number of blades.

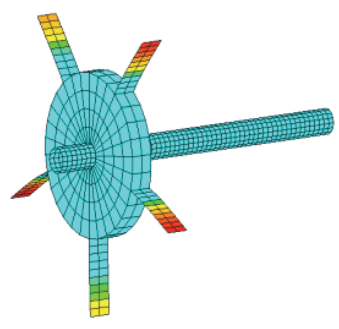

(a) Mode of blade (at $1 / 6)$

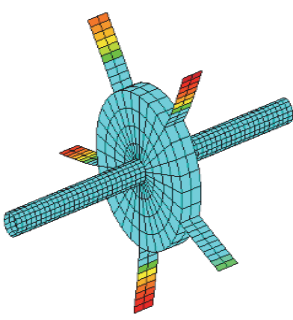

(b) Mode of blade (at 1/2)

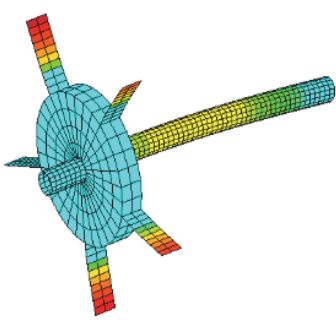

(c) Mode of rotor (at 1/6)

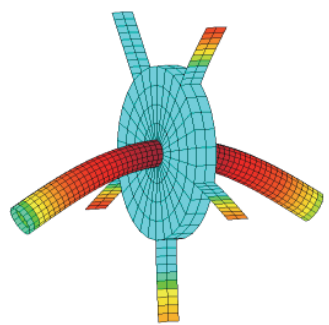

(d) Mode of rotor (at 1/2)

FIGURE 21: Mode shapes of rotor-blade coupling system due to the position of disk.

obvious difference between the mode shape of SB mode and $\mathrm{BB}$ mode of blade; that is to say it is difficult to distinguish these two modes through their mode shapes. Furthermore, it is also revealed the shaft does not bend obviously in the mode of blade but the blade bends quite obviously in the mode of rotor. Therefore, a conclusion is drawn that the mode of blade has no effect on the deformation of rotor but the mode of rotor has an obvious effect on the deformation of blade.

Based on the conclusion that has been drawn, when discussing the influence of different factors on the mode shape of coupling system, the mode shape of blade is represented by the first-order mode shape of SB mode. Similarly, the mode shape of rotor is represented by the first-order mode shape of rotor.

4.1. The Influence of Blades Number on Mode Shapes. Mode shapes of rotor-blade coupling system changes due to the number of blades are shown in Figure 20. Figure 20(a) is blade mode shape of rotor-blade coupling system with 4 blades; Figure 20(b) is blade mode shape of rotor-blade coupling system with 4 blades. Figure 20 (c) is rotor mode shape of rotor-blade coupling system with 4 blades; and Figure 20(d) is rotor mode shape of rotor-blade coupling system with 7 blades. It could be found that there are no obvious changes of blade and shaft bending deformations with the increase of the number of blades. This shows that the number of blades has no significant effect on the mode shape of blade and rotor.

4.2. The Influence of Disk Position on Mode Shapes. Figure 21 illustrates how the mode shape of rotor-blade coupling system changes due to the position of disk. Figure 21(a) is blade mode shape of rotor-blade coupling system when the position of disk is 1/6; Figure 21(b) is blade mode shape of rotor-blade coupling system when the position of disk is $1 / 2$. Figure $21(c)$ is rotor mode shape of rotor-blade coupling 


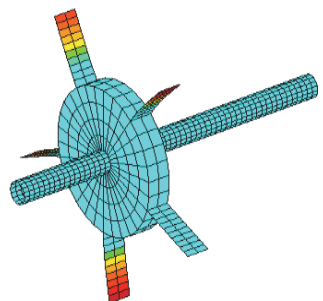

(a) Mode of blade (100 rad/s)

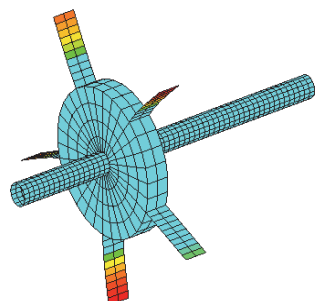

(b) Mode of blade $(1000 \mathrm{rad} / \mathrm{s})$

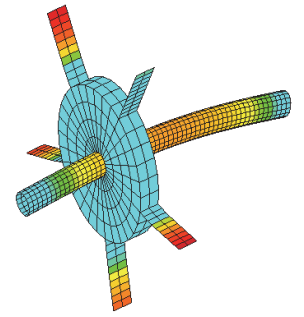

(c) Mode of rotor $(100 \mathrm{rad} / \mathrm{s})$

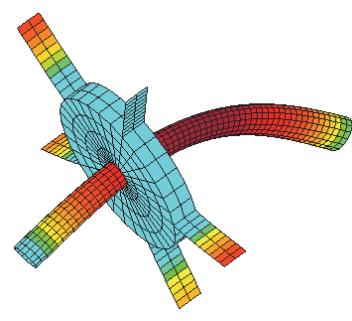

(d) Mode of rotor (1000 rad/s)

FIGURE 22: Mode shapes of rotor-blade coupling system due to the rotational speed.

system when the position of disk is 1/6; Figure 21(d) is rotor mode shape of rotor-blade coupling system when the position of disk is $1 / 2$. It could be found that when the disk moves from the end to the center of shaft, the bending deformation of blade remains almost unchanged but that of shaft increases obviously. This shows that the position of disk has an obvious effect on the mode shape of rotor.

4.3. The Influence of Rotational Speed on Mode Shapes. Figure 22 shows the mode shape of rotor-blade coupling system change due to the rotational speed. Figure 22 is blade mode shape of rotor-blade coupling system when the rational speed is $100 \mathrm{rad} / \mathrm{s}$; Figure 22(b) is blade mode shape of rotorblade coupling system when the rational speed is $1000 \mathrm{rad} / \mathrm{s}$. Figure 22(c) is rotor mode shape of rotor-blade coupling system when the rational speed is $100 \mathrm{rad} / \mathrm{s}$; Figure 22(d) is rotor mode shape of rotor-blade coupling system when the rational speed is $1000 \mathrm{rad} / \mathrm{s}$. It could be found that, with the increase of rotational speed, the bending deformation of blade remains almost unchanged but that of shaft increases obviously. This shows that the position of disk has an obvious effect on the mode shape of rotor.

\section{Conclusion}

The model of rotor-blade coupling system model is established, the mode function of elastic support beam is deduced, the differential equation of system is deduced by the semianalytical method, and the influences of blades number, disk position, and support stiffness on system characteristic value, Campbell diagram, and mode shape of vibration are studied by numerical simulation.

(1) Because of the coupling between shaft and blade, there are SB and BB modes dominated by blade's first two-order modes which could be found and the BB modes are of repeated frequencies of $\left(N_{b}-\right.$ 2) multiplicity and the SB modes are of repeated frequencies of (2) multiplicity. Frequencies of both modes could be found in the Campbell diagram of blade all the time.

(2) With the increase of the number of blades, the frequency of SB mode increases linearly, that of BB mode is constant, and that of rotor decreases linearly.
The changing trend of Campbell diagram is the same as natural frequency.

(3) Frequencies of SB mode of blade and rotor mode are affected by the position of disk and change symmetrically with the center of shaft. What is more, the changing trend of first- and second-order frequency is different. But that of Campbell diagram is the same as natural frequency.

(4) It could be found that there is no obvious difference between the mode shape of SB mode and BB mode of blade. Furthermore, the mode of blade has no effect on the deformation of rotor but the mode of rotor has an obvious effect on the deformation of blade. The number of blades has no significant effect on the mode shape of blade and rotor but the position of disk and rotational speed have an obvious effect on the mode shape of rotor.

\section{Appendix}

\section{A. Vectors and Matrices Related to the Rotor System}

(1) Expression of discrete kinetic energy of shaft can be shown as follows:

$$
\begin{aligned}
T_{s} & =\int_{0}^{L_{s}} \frac{1}{2} \rho A\left(\dot{\boldsymbol{\eta}}^{T}(t) \mathbf{X}^{T}(z) \mathbf{X}(z) \dot{\boldsymbol{\eta}}(t)\right. \\
& \left.+\dot{\boldsymbol{\xi}}^{T}(t) \mathbf{Y}^{T}(z) \mathbf{Y}(z) \dot{\boldsymbol{\xi}}(t)\right)+\frac{1}{2} J_{p} \dot{\boldsymbol{\phi}}^{2}+\frac{1}{2} \\
& \cdot J_{d}\left(\left[\mathbf{X}^{\prime}(z) \dot{\boldsymbol{\eta}}(t)+\frac{E I}{\kappa A G} \mathbf{X}^{\prime \prime \prime}(z) \dot{\boldsymbol{\eta}}(t)\right]^{T}\right. \\
& \left.\cdot\left[\mathbf{X}^{\prime}(z) \dot{\boldsymbol{\eta}}(t)+\frac{E I}{\kappa A G} \mathbf{X}^{\prime \prime \prime}(z) \dot{\boldsymbol{\eta}}(t)\right]\right)+\frac{1}{2} \\
& \cdot J_{d}\left[\mathbf{Y}^{\prime}(z) \dot{\boldsymbol{\xi}}(t)+\frac{E I}{\kappa A G} \mathbf{Y}^{\prime \prime \prime}(z) \dot{\boldsymbol{\xi}}(t)\right]^{T}\left[\mathbf{Y}^{\prime}(z)\right. \\
& \left.\cdot \dot{\boldsymbol{\xi}}(t)+\frac{E I}{\kappa A G} \mathbf{Y}^{\prime \prime \prime}(z) \dot{\boldsymbol{\xi}}(t)\right]+\frac{1}{2} J_{p} \dot{\phi}\left(\left[\mathbf{X}^{\prime}(z) \dot{\boldsymbol{\eta}}(t)\right.\right.
\end{aligned}
$$




$$
\begin{aligned}
& \left.+\frac{E I}{\kappa A G} \mathbf{X}^{\prime \prime \prime}(z) \dot{\boldsymbol{\eta}}(t)\right]^{T}\left[\mathbf{Y}^{\prime}(z) \boldsymbol{\xi}(t)\right. \\
& \left.\left.+\frac{E I}{\kappa A G} \mathbf{Y}^{\prime \prime \prime}(z) \xi(t)\right]\right)-\frac{1}{2} J_{p} \dot{\boldsymbol{\phi}}\left(\left[\mathbf{X}^{\prime}(z) \boldsymbol{\eta}(t)\right.\right. \\
& \left.+\frac{E I}{\kappa A G} \mathbf{X}^{\prime \prime \prime}(z) \boldsymbol{\eta}(t)\right]^{T}\left[\mathbf{Y}^{\prime}(z) \dot{\boldsymbol{\xi}}(t)\right. \\
& \left.\left.+\frac{E I}{\kappa A G} \mathbf{Y}^{\prime \prime \prime}(z) \dot{\boldsymbol{\xi}}(t)\right]\right)+\frac{1}{2} I_{s} \int_{0}^{L_{s}} \dot{\mathbf{q}}_{\theta}^{\mathrm{T}} \boldsymbol{\Phi}^{\mathrm{T}} \boldsymbol{\Phi} \dot{\mathbf{q}}_{\theta} \mathrm{d} z .
\end{aligned}
$$

(2) Expression of discrete kinetic energy of disk can be expressed as

$$
\begin{aligned}
& T_{d} \\
& =\frac{1}{2} \dot{\boldsymbol{\eta}}^{T}(t) m_{d} \mathbf{X}^{T}\left(z_{d}\right) \mathbf{X}\left(z_{d}\right) \dot{\boldsymbol{\eta}}(t) \\
& +\frac{1}{2} \dot{\xi}^{T}(t) m_{d} \mathbf{Y}^{T}\left(z_{d}\right) \mathbf{Y}\left(z_{d}\right) \dot{\xi}(t) \\
& +\frac{1}{2} \dot{\boldsymbol{\eta}}^{T}(t) J_{d z} \frac{E I}{\kappa A G} \mathbf{X}^{\prime \prime \prime T}\left(z_{d}\right) \mathbf{X}^{\prime}\left(z_{d}\right) \dot{\boldsymbol{\eta}}(t) \\
& +\frac{1}{2} \dot{\boldsymbol{\eta}}^{T}(t) J_{d z}\left(\frac{E I}{\kappa A G}\right)^{2} \mathbf{X}^{\prime \prime \prime T}\left(z_{d}\right) \mathbf{X}^{\prime \prime \prime}\left(z_{d}\right) \dot{\boldsymbol{\eta}}(t) \\
& +\frac{1}{2} \dot{\xi}^{T}(t) J_{d z} \mathbf{Y}^{\prime T}\left(z_{d}\right) \mathbf{Y}^{\prime}\left(z_{d}\right) \dot{\xi}(t) \\
& +\frac{1}{2} \dot{\xi}^{T}(t) J_{d z} \frac{E I}{\kappa A G} \mathbf{Y}^{\prime T}\left(z_{d}\right) \mathbf{Y}^{\prime \prime \prime}\left(z_{d}\right) \dot{\xi}(t) \\
& +\frac{1}{2} \dot{\xi}^{T}(t) J_{d z} \frac{E I}{\kappa A G} \mathbf{Y}^{\prime \prime \prime T}\left(z_{d}\right) \mathbf{Y}^{\prime}\left(z_{d}\right) \dot{\xi}(t) \\
& +\frac{1}{2} \dot{\xi}^{T}(t) J_{d z}\left(\frac{E I}{\kappa A G}\right)^{2} \mathbf{Y}^{\prime \prime \prime T}\left(z_{d}\right) \mathbf{Y}^{\prime \prime \prime}\left(z_{d}\right) \dot{\xi}(t) \\
& +\frac{1}{2} \dot{\boldsymbol{\eta}}^{T}(t) J_{p} \dot{\phi} \mathbf{X}^{\prime T}\left(z_{d}\right) \mathbf{Y}^{\prime}\left(z_{d}\right) \boldsymbol{\xi}(t) \\
& +\frac{1}{2} \dot{\boldsymbol{\eta}}^{T}(t) J_{z} \dot{\phi} \frac{E I}{\kappa A G} \mathbf{X}^{\prime T}\left(z_{d}\right) \mathbf{Y}^{\prime \prime \prime}\left(z_{d}\right) \boldsymbol{\xi}(t) \\
& +\frac{1}{2} \dot{\boldsymbol{\eta}}^{T}(t) J_{z} \dot{\phi} \frac{E I}{\kappa A G} \mathbf{X}^{\prime \prime \prime T}\left(z_{d}\right) \mathbf{Y}^{\prime}\left(z_{d}\right) \boldsymbol{\xi}(t) \\
& +\frac{1}{2} \dot{\boldsymbol{\eta}}^{T}(t) J_{z} \dot{\phi}\left(\frac{E I}{\kappa A G}\right)^{2} \mathbf{X}^{\prime \prime \prime T}\left(z_{d}\right) \mathbf{Y}^{\prime \prime \prime}\left(z_{d}\right) \xi(t) \\
& -\frac{1}{2} \boldsymbol{\eta}^{T}(t) J_{z} \dot{\phi} \mathbf{X}^{\prime T}\left(z_{d}\right) \mathbf{Y}^{\prime}\left(z_{d}\right) \dot{\xi}(t) \\
& -\frac{1}{2} \boldsymbol{\eta}^{T}(t) J_{z} \dot{\phi} \frac{E I}{\kappa A G} \mathbf{X}^{\prime T}\left(z_{d}\right) \mathbf{Y}^{\prime \prime \prime}\left(z_{d}\right) \dot{\xi}(t) \\
& -\frac{1}{2} \boldsymbol{\eta}^{T}(t) J_{z} \dot{\phi} \frac{E I}{\kappa A G} \mathbf{X}^{\prime \prime \prime T}\left(z_{d}\right) \mathbf{Y}^{\prime}\left(z_{d}\right) \dot{\boldsymbol{\xi}}(t) \\
& -\frac{1}{2} \boldsymbol{\eta}^{T}(t) J_{z} \dot{\phi}\left(\frac{E I}{\kappa A G}\right)^{2} \mathrm{X}^{\prime \prime \prime T}\left(z_{d}\right) \mathrm{Y}^{\prime \prime \prime}\left(z_{d}\right) \dot{\xi}(t) \text {. }
\end{aligned}
$$

(3) Expression of discrete potential energy of shaft can be written as

$$
\begin{aligned}
U_{s} & =\frac{1}{2} \int_{0}^{L_{s}}\left\{E I \left(\left[\mathbf{X}^{\prime \prime}(z) \boldsymbol{\eta}(t)\right]^{T}\left[\mathbf{X}^{\prime \prime}(z) \boldsymbol{\eta}(t)\right]\right.\right. \\
& \left.+\left[\mathbf{Y}^{\prime \prime}(z) \boldsymbol{\xi}(t)\right]^{T}\left[\mathbf{Y}^{\prime \prime}(z) \boldsymbol{\xi}(t)\right]\right) \\
& +\kappa G A\left(\left[\frac{E I}{\kappa A G} \mathbf{X}^{\prime \prime \prime}(z) \boldsymbol{\eta}(t)\right]^{T}\right. \\
& \cdot\left[\frac{E I}{\kappa A G} \mathbf{X}^{\prime \prime \prime}(z) \boldsymbol{\eta}(t)\right]+\left[\frac{E I}{\kappa A G} \mathbf{Y}^{\prime \prime \prime}(z) \boldsymbol{\xi}(t)\right]^{T} \\
& \left.\left.\cdot\left[\frac{E I}{\kappa A G} \mathbf{Y}^{\prime \prime \prime \prime}(z) \boldsymbol{\xi}(t)\right]\right)\right\} d z=\frac{1}{2} \\
& \cdot \int_{0}^{L_{s}}\left\{\mathbf{q}_{\theta}^{T} G_{s} J_{s} \Phi^{\prime T} \Phi^{\prime} \mathbf{q}_{\theta}+\boldsymbol{\eta}^{T}(t) E I \mathbf{X}^{\prime \prime T}(z) \mathbf{X}^{\prime \prime}(z)\right. \\
& \cdot \boldsymbol{\eta}(t)+\xi^{T}(t) E I \mathbf{Y}^{\prime \prime T}(z) \mathbf{Y}^{\prime \prime}(z) \boldsymbol{\xi}(t)+\boldsymbol{\eta}^{T}(t) \\
& \cdot \frac{(E I)^{2}}{\kappa A G} \mathbf{X}^{\prime \prime \prime T}(z) \mathbf{X}^{\prime \prime \prime}(z) \boldsymbol{\eta}(t)+\boldsymbol{\xi}^{T}(t) \frac{(E I)^{2}}{\kappa A G} \\
& \left.\cdot \mathbf{Y}^{\prime \prime \prime \prime}(z) \mathbf{Y}^{\prime \prime \prime}(z) \boldsymbol{\xi}(t)\right\} \mathrm{d} z .
\end{aligned}
$$

(4) The specific meaning of each parameter in the vibration differential equation of rotor is

$$
\begin{aligned}
\mathbf{M}_{s 1} & =\int_{0}^{L_{s}}\left[\rho A \mathbf{X}^{T}(z) \mathbf{X}(z)+J_{d} \mathbf{X}^{\prime T}(z) \mathbf{X}^{\prime}(z)\right. \\
& +J_{d} \frac{E I}{\kappa A G} \mathbf{X}^{\prime T}(z) \mathbf{X}^{\prime \prime \prime}(z) \\
& +J_{d} \frac{E I}{\kappa A G} \mathbf{X}^{\prime \prime \prime T}(z) \mathbf{X}^{\prime}(z) \\
& \left.+J_{d}\left(\frac{E I}{\kappa A G}\right)^{2} \mathbf{X}^{\prime \prime \prime T}(z) \mathbf{X}^{\prime \prime \prime}(z)\right] \mathrm{d} z \\
\mathbf{M}_{s 2} & =\int_{0}^{L_{s}}\left[\rho A \mathbf{Y}^{T}(z) \mathbf{Y}(z)+J_{d} \mathbf{Y}^{\prime T}(z) \mathbf{Y}^{\prime}(z)\right. \\
& \left.+J_{d} \frac{E I}{\kappa A G} \mathbf{Y}^{\prime T}(z) \mathbf{Y}^{\prime \prime \prime}(z)\right]+J_{d} \frac{E I}{\kappa A G} \mathbf{Y}^{\prime \prime \prime T}(z) \\
& +\mathbf{Y}^{\prime}(z)+J_{d}\left(\frac{E I}{\kappa A G}\right)^{2} \mathbf{Y}^{\prime \prime \prime T}(z) \mathbf{Y}^{\prime \prime \prime}(z) \mathrm{d} z \\
M_{\theta} & =I_{s} \int_{0}^{L_{s}} \mathbf{\Phi}^{T} \mathbf{\Phi} d z \\
\mathbf{G}_{s 1} & =\int_{0}^{L_{s}}\left[J_{p} \mathbf{X}^{\prime T}(z) \mathbf{Y}^{\prime}(z)\right. \\
& +J_{p} \frac{E I}{\kappa A G} \mathbf{X}^{\prime T}(z) \mathbf{Y}^{\prime \prime \prime}(z)+J_{p} \frac{E I}{\kappa A G} \mathbf{X}^{\prime \prime \prime T}(z) \mathbf{Y}^{\prime}(z)
\end{aligned}
$$




$$
\begin{aligned}
& \left.+J_{p}\left(\frac{E I}{\kappa A G}\right)^{2} \mathbf{X}^{\prime \prime \prime T}(z) \mathbf{Y}^{\prime \prime \prime}(z)\right] \mathrm{d} z \\
& \mathbf{G}_{s 2}=\int_{0}^{L_{s}}\left[-J_{p}\left(\frac{E I}{\kappa A G}\right)^{2} \mathbf{Y}^{\prime \prime \prime T}(z) \mathbf{X}^{\prime \prime \prime}(z)\right. \\
& -J_{p} \frac{E I}{\kappa A G} \mathbf{Y}^{\prime \prime \prime T}(z) \mathbf{X}^{\prime}(z)-J_{p} \frac{E I}{\kappa A G} \mathbf{Y}^{\prime T}(z) \mathbf{X}^{\prime \prime \prime}(z) \\
& \left.-J_{p} \mathbf{Y}^{\prime T}(z) \mathbf{X}^{\prime}(z)\right] \mathrm{d} z \\
& \mathbf{M}_{d 1}=m_{d} \mathbf{X}^{T}\left(z_{d}\right) \mathbf{X}\left(z_{d}\right)+J_{d} \mathbf{X}^{\prime T}\left(z_{d}\right) \mathbf{X}^{\prime}\left(z_{d}\right)+J_{d} \\
& \cdot \frac{E I}{\kappa A G} \mathbf{X}^{\prime T}\left(z_{d}\right) \mathbf{X}^{\prime \prime \prime}\left(z_{d}\right)+J_{d} \frac{E I}{\kappa A G} \mathbf{X}^{\prime \prime \prime T}\left(z_{d}\right) \\
& \cdot \mathbf{X}^{\prime}\left(z_{d}\right)+J_{d}\left(\frac{E I}{\kappa A G}\right)^{2} \mathbf{X}^{\prime \prime \prime T}\left(z_{d}\right) \mathbf{X}^{\prime \prime \prime}\left(z_{d}\right) \\
& \mathbf{M}_{d 2}=m_{d} \mathbf{Y}^{T}\left(z_{d}\right) \mathbf{Y}\left(z_{d}\right)+J_{d} \mathbf{Y}^{T}\left(z_{d}\right) \mathbf{Y}^{\prime}\left(z_{d}\right)+J_{d} \\
& \cdot \frac{E I}{\kappa A G} \mathbf{Y}^{\prime T}\left(z_{d}\right) \mathbf{Y}^{\prime \prime \prime}\left(z_{d}\right)+J_{d} \frac{E I}{\kappa A G} \mathbf{Y}^{\prime \prime \prime T}\left(z_{d}\right) \\
& \cdot \mathbf{Y}^{\prime}\left(z_{d}\right)+J_{d}\left(\frac{E I}{\kappa A G}\right)^{2} \mathbf{Y}^{\prime \prime \prime T}\left(z_{d}\right) \mathbf{Y}^{\prime \prime \prime}\left(z_{d}\right) \\
& \mathbf{G}_{d 1}=J_{z} \frac{E I}{\kappa A G} \mathbf{X}^{\prime \prime \prime T}\left(z_{d}\right) \mathbf{Y}^{\prime}\left(z_{d}\right)+J_{z}\left(\frac{E I}{\kappa A G}\right)^{2} \\
& \cdot \mathbf{X}^{\prime \prime \prime T}\left(z_{d}\right) \mathbf{Y}^{\prime \prime \prime}\left(z_{d}\right)+J_{z} \mathbf{X}^{\prime T}\left(z_{d}\right) \mathbf{Y}^{\prime}\left(z_{d}\right)+J_{z} \\
& \cdot \frac{E I}{\kappa A G} \mathbf{X}^{\prime T}\left(z_{d}\right) \mathbf{Y}^{\prime \prime \prime}\left(z_{d}\right) \\
& \mathbf{G}_{d 2}=-J_{z}\left(\frac{E I}{\kappa A G}\right)^{2} \mathbf{Y}^{\prime \prime \prime T}\left(z_{d}\right) \mathbf{X}^{\prime \prime \prime}\left(z_{d}\right)-J_{z} \frac{E I}{\kappa A G} \\
& \cdot \mathbf{Y}^{\prime \prime \prime T}\left(z_{d}\right) \mathbf{X}^{\prime}\left(z_{d}\right)-J_{z} \frac{E I}{\kappa A G} \mathbf{Y}^{\prime T}\left(z_{d}\right) \mathbf{X}^{\prime \prime \prime}\left(z_{d}\right) \\
& -J_{z} \mathbf{Y}^{\prime T}\left(z_{d}\right) \mathbf{X}^{\prime}\left(z_{d}\right) \\
& \mathbf{K}_{s 1}=\int_{0}^{L_{s}}\left[E I \mathbf{X}^{\prime \prime T}(z) \mathbf{X}^{\prime \prime}(z)\right. \\
& \left.+\left(\frac{E I}{\kappa A G}\right)^{2} \mathbf{X}^{\prime \prime \prime T}(z) \mathbf{X}^{\prime \prime \prime}(z)\right] \mathrm{d} z \\
& \mathbf{K}_{s 2}=\int_{0}^{L_{s}}\left[E I \mathbf{Y}^{\prime \prime T}(z) \mathbf{Y}^{\prime \prime}(z)\right. \\
& \left.+\left(\frac{E I}{\kappa A G}\right)^{2} \mathrm{Y}^{\prime \prime \prime T}(z) \mathrm{Y}^{\prime \prime \prime}(z)\right] \mathrm{d} z \\
& \mathbf{K}_{\boldsymbol{\theta}}=G_{s} J_{s} \int_{0}^{L_{s}} \Phi^{\prime T} \Phi^{\prime} \mathrm{d} z .
\end{aligned}
$$

\section{B. Vectors and Matrices Related to the Blade}

(1) The specific meaning of each transformation matrix of blade can be obtained as follows:

$$
\begin{aligned}
& \mathbf{A}_{\mathbf{0}}=\left[\begin{array}{ccc}
\cos \theta_{T} & -\sin \theta_{T} & 0 \\
\sin \theta_{T} & \cos \theta_{T} & 0 \\
0 & 0 & 1
\end{array}\right] \\
& \mathbf{A}_{\mathbf{1}}=\left[\begin{array}{ccc}
\cos \psi & -\sin \psi & 0 \\
\sin \psi & \cos \psi & 0 \\
0 & 0 & 1
\end{array}\right] \\
& \mathbf{A}_{\mathbf{2}}=\left[\begin{array}{ccc}
1 & 0 & 0 \\
0 & \cos \theta_{y} & -\sin \theta_{y} \\
0 & \sin \theta_{y} & \cos \theta_{y}
\end{array}\right] \\
& \mathbf{A}_{3}=\left[\begin{array}{ccc}
\cos \theta_{x} & 0 & \sin \theta_{x} \\
0 & 1 & 0 \\
-\sin \theta_{x} & 0 & \cos \theta_{x}
\end{array}\right],
\end{aligned}
$$

where $\psi=\Omega t+(i-1) 2 \pi / N_{b}$ and $(i-1) 2 \pi / N_{b}$ indicates the position of the $i$ th blade in the blade, $N_{b}$ is the number of blades, $\theta_{T}$ is the twist angle at disk hub, and $\theta_{x}$ and $\theta_{y}$ are swinging angles of disk.

\section{Vectors and Matrices Related to the Rotor-Blade Coupling System}

(1) $q_{r b}$ is generalized coordinate vector of rotor-blade coupling system.

$$
q_{r b}=\left[\begin{array}{lll}
q_{r} & q_{\theta} & q_{b}
\end{array}\right]^{T},
$$

where $q_{r}$ and $q_{\theta}$ are vectors of translational and torsional degrees of freedom of rotor; $q_{b}$ is the vector of degrees of freedom of blade.

(2) $M_{r b}$ is mass matrix of rotor-blade coupling system.

$$
M_{r b}=\left[\begin{array}{ccc}
M_{r} & 0 & M_{s b} \\
0 & M_{\theta} & M_{\theta b} \\
M_{s b}^{T} & M_{\theta b}^{T} & M_{b}
\end{array}\right],
$$

where $M_{r}$ and $M_{\theta}$ are vectors of translational and torsional mass matrix of rotor; $M_{b}$ is the vector of mass matrix of blade; $M_{\theta b}$ and $M_{s b}$ are coupling mass matrix of system.

(3) $C_{r b}$ is damping matrix of rotor-blade system (including proportional damping and gyro matrix)

$$
C_{r b}=\left[\begin{array}{ccc}
C_{r} & C_{s \theta} & C_{s b} \\
C_{s \theta}^{T} & C_{\theta} & C_{\theta b} \\
C_{s b}^{T} & C_{\theta b}^{T} & C_{b}
\end{array}\right],
$$

where $C_{r}$ and $C_{\theta}$ are vectors of translational and torsional damping matrix of rotor; $C_{b}$ is the vector of damping matrix 
of blade; $C_{s \theta}, C_{s b}$, and $C_{\theta b}$ are coupling damping matrix of system.

(4) $K_{r b}$ is stiffness matrix of rotor-blade coupling system

$$
K_{r b}=\left[\begin{array}{ccc}
K_{r} & 0 & 0 \\
0 & K_{\theta} & 0 \\
0 & 0 & K_{b}
\end{array}\right],
$$

where $K_{r}$ and $K_{\theta}$ are vectors of translational and torsional stiffness matrix of rotor; $K_{b}$ is the vector of stiffness matrix of blade.

\section{Geometric and Material Properties of Rotor-Blade System}

\author{
Shaft \\ $\rho_{s}:$ Density $\left(7850 \mathrm{~kg} / \mathrm{m}^{3}\right)$ \\ $G_{s}$ : Shear modulus (75 Gpa) \\ $l_{s}$ : Shaft length $(0.6 \mathrm{~m})$ \\ $R_{s}$ : Radius $(0.015 \mathrm{~m})$. \\ Disk \\ $\rho_{d}:$ Density $\left(7850 \mathrm{~kg} / \mathrm{m}^{3}\right)$ \\ $E_{d}$ : Young's modulus $(200 \mathrm{Gpa})$ \\ $R_{d}$ : Outer radius $(0.2 \mathrm{~m})$ \\ $B_{d}$ : Thickness $(0.015 \mathrm{~m})$ \\ $\mu_{d}$ : Poisson ratio (0.3). \\ Blade

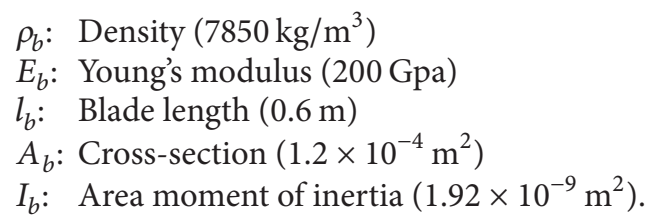

\section{Competing Interests}

The authors declare that they have no competing interests.

\section{Acknowledgments}

The project is supported by the China Natural Science Funds (no. 51575093) and Fundamental Research Funds for the Central Universities (nos. N140304002 and N140301001).

\section{References}

[1] R. G. Parker and C. D. Mote, "Exact perturbation for the vibration of almost annular or circular plates," Journal of Vibration and Acoustics, Transactions of the ASME, vol. 118, no. 3, pp. 436-445, 1996.

[2] R. M. H. Khorasany and S. G. Hutton, "An analytical study on the effect of rigid body translational degree of freedom on the vibration characteristics of elastically constrained rotating disks," International Journal of Mechanical Sciences, vol. 52, no. 9, pp. 1186-1192, 2010.
[3] M. Kim, J. Moon, and J. A. Wickert, "Spatial modulation of repeated vibration modes in rotationally periodic structures," Journal of Vibration and Acoustics, Transactions of the ASME, vol. 122, no. 1, pp. 62-68, 2000.

[4] J. A. Dopkin and T. E. Shoup, "Rotor resonant speed reduction caused by flexibility of disks," Journal of Engineering for Industry ASME, vol. 96, no. 4, pp. 1328-1333, 1974.

[5] N. Anegawa, H. Fujiwara, and O. Matsushita, "Resonance and instability of blade-shaft coupled bending vibrations," International Journal of Fluid Machinery \& Systems, vol. 1, no. 1, pp. 169-180, 2008.

[6] Ö. Turhan and G. Bulut, "Linearly coupled shaft-torsional and blade-bending vibrations in multi-stage rotor-blade systems," Journal of Sound and Vibration, vol. 296, no. 1-2, pp. 292-318, 2006.

[7] Y.-J. Chiu and D.-Z. Chen, "The coupled vibration in a rotating multi-disk rotor system," International Journal of Mechanical Sciences, vol. 53, no. 1, pp. 1-10, 2011.

[8] N. Lesaffre, J.-J. Sinou, and F. Thouverez, "Contact analysis of a flexible bladed-rotor," European Journal of Mechanics, A/Solids, vol. 26, no. 3, pp. 541-557, 2007.

[9] S. C. Huang and K. B. Ho, "Coupled shaft-torsion and bladebending vibrations of a rotating shaft-disk-blade unit," Journal of Engineering for Gas Turbines and Power, vol. 118, no. 1, pp. 100-106, 1996.

[10] Y. Chiu and C.-H. Yang, "The coupled vibration in a rotating multi-disk rotor system with grouped blades," Journal of Mechanical Science and Technology, vol. 28, no. 5, pp. 1653-1662, 2014.

[11] V. Omprakash and V. Ramamurti, "Coupled free vibration characteristics of rotating tuned bladed disk systems," Journal of Sound and Vibration, vol. 140, no. 3, pp. 413-435, 1990.

[12] F. Sisto, A. Chang, and M. Sutcu, "Influence of coriolis forces on gyroscopic motion of spinning blades," Journal of engineering for power, vol. 105, no. 2, pp. 342-347, 1983.

[13] D. J. Ewins, "Vibration characteristics of bladed disc assemblies," Journal of Mechanical Engineering Science, vol. 15, no. 3, pp. 165186, 1973.

[14] F. Kushner, "Disc vibration — rotating blade and stationary vane interaction," Journal of mechanical design, vol. 102, no. 3, pp. 579-584, 1980.

[15] S.-B. Chun and C.-W. Lee, "Vibration analysis of shaft-bladed disk system by using substructure synthesis and assumed modes method," Journal of Sound \& Vibration, vol. 189, no. 5, pp. 587608, 1996.

[16] C.-H. Yang and S.-C. Huang, "The coupled vibration in a shaftdisk-blades system," Journal of the Chinese Institute of Engineers, vol. 28, no. 1, pp. 89-99, 2005.

[17] C.-H. Yang and S.-C. Huang, "The coupled vibration in a shaft-disk-blades system," Journal of the Chinese Institute of Engineers, vol. 28, no. 1, pp. 89-99, 2005.

[18] C.-H. Yang and S.-C. Huang, "The influence of disk's flexibility on coupling vibration of shaft-disk-blades systems," Journal of Sound and Vibration, vol. 301, no. 1-2, pp. 1-17, 2007.

[19] C.-H. Yang and S.-C. Huang, "Coupling vibrations in rotating shaft-disk-blades system," Journal of Vibration and Acoustics, Transactions of the ASME, vol. 129, no. 1, pp. 48-57, 2007.

[20] Y.-J. Chiu and S.-C. Huang, "The influence on coupling vibration of a rotor system due to a mistuned blade length," International Journal of Mechanical Sciences, vol. 49, no. 4, pp. 522-532, 2007. 


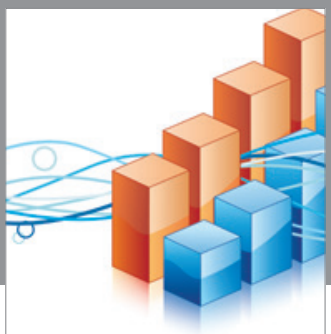

Advances in

Operations Research

vatem alat4

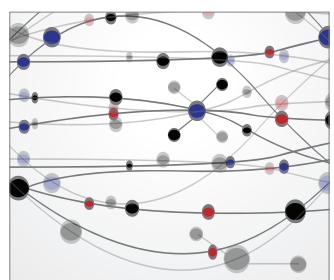

\section{The Scientific} World Journal
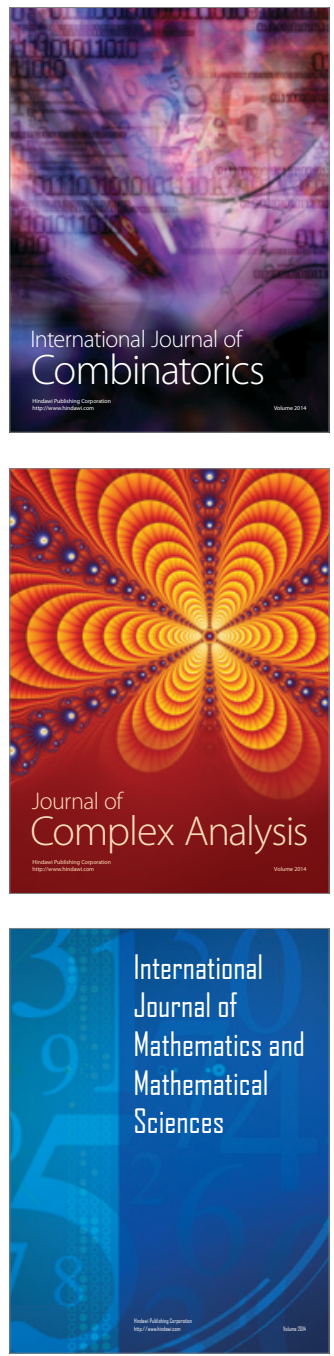
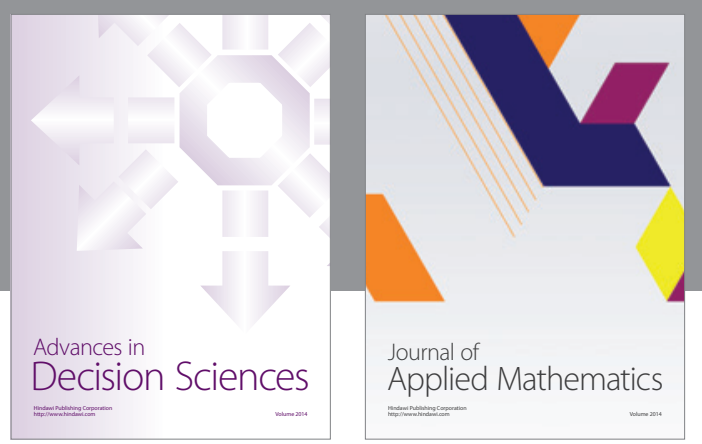

Algebra

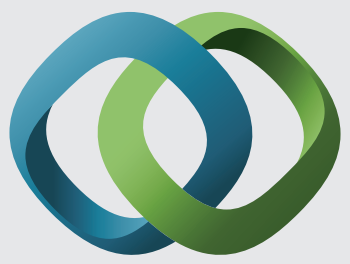

\section{Hindawi}

Submit your manuscripts at

https://www.hindawi.com
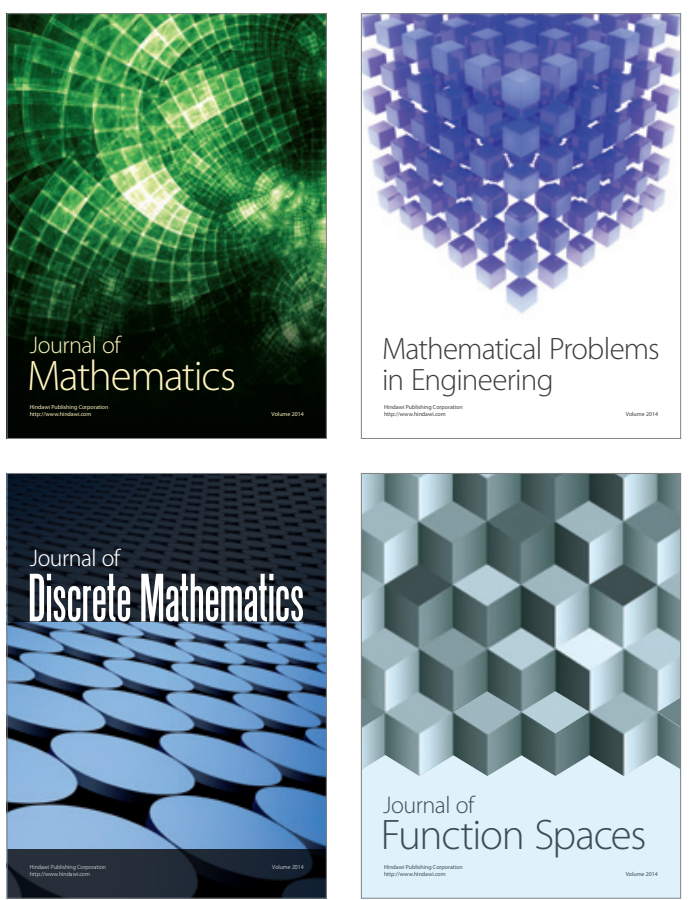

Mathematical Problems in Engineering
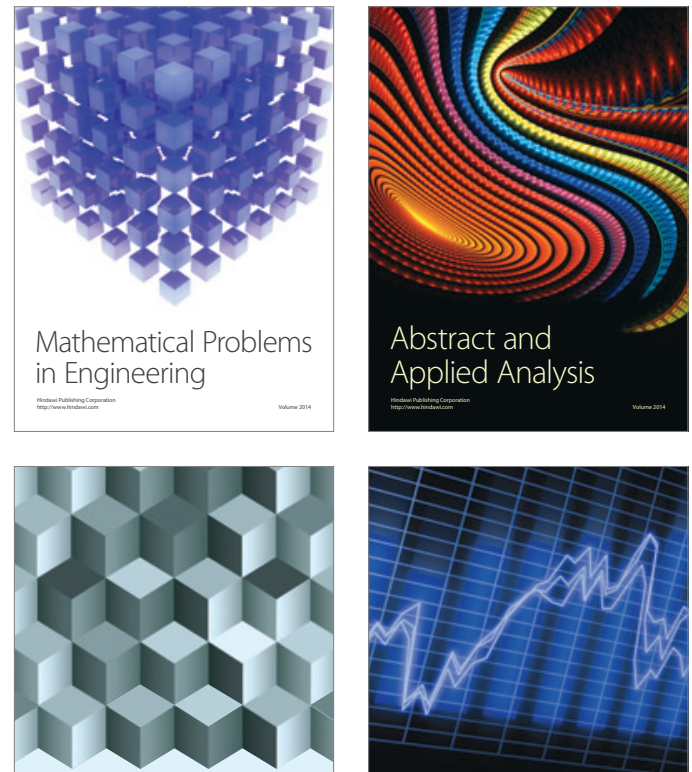

Journal of

Function Spaces

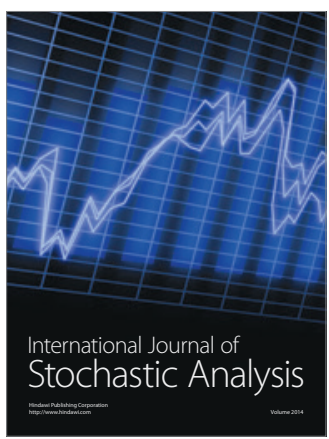

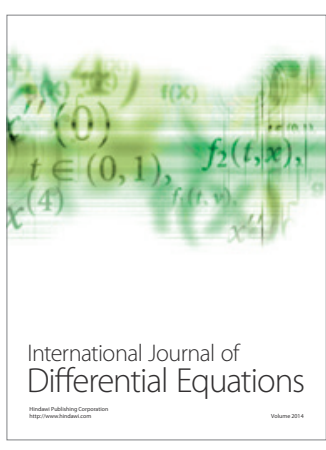
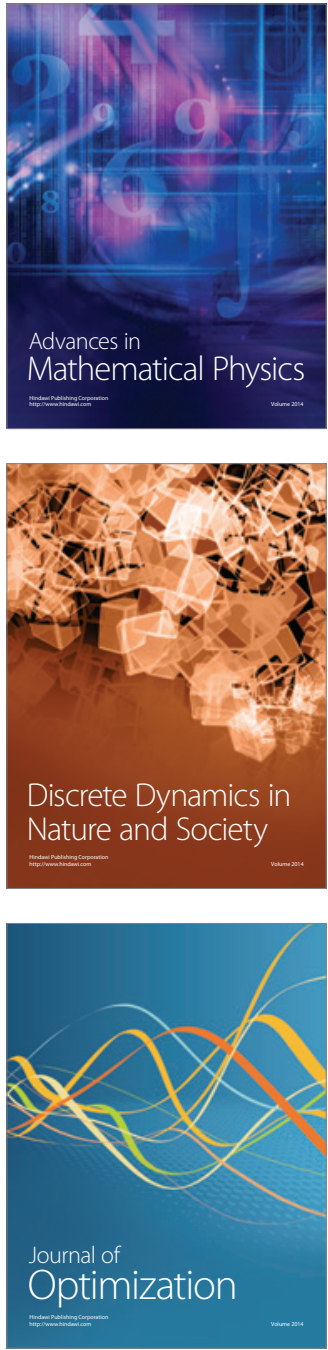